\title{
A vertex-based finite volume method applied to non-linear material problems in computational solid mechanics
}

\author{
G. A. Taylor*, C. Bailey and M. Cross \\ Centre for Numerical Modelling and Process Analysis, University of Greenwich, London SE10 9JH, UK.
}

\begin{abstract}
SUMMARY
A vertex-based Finite Volume (FV) method is presented for the computational solution of quasi-static solid mechanics problems involving material non-linearity and infinitesimal strains. The problems are analysed numerically with fully unstructured meshes that consist of a variety of two and three dimensional element types. A detailed comparison between the vertex-based FV and the standard Galerkin Finite Element (FE) methods is provided with regard to discretisation, solution accuracy and computational efficiency. For some problem classes a direct equivalence of the two methods is demonstrated, both theoretically and numerically. However, for other problems some interesting advantages and disadvantages of the FV formulation over the Galerkin FE method are highlighted. Copyright (c) 2000 John Wiley \& Sons, Ltd.
\end{abstract}

KEY WORDS: Finite Volume; vertex-based; Computational Solid Mechanics

\footnotetext{
${ }^{*}$ Correspondence to: Brunel Institute of Power Systems, Brunel University, Middlesex UB8 3PH, UK.

E-mail: Gareth.Taylor@brunel.ac.uk, Tel: (44/0)1895 274000 ext 2773, Fax: (44/0)1895 258728
}

Received 17 October 2000 


\section{INTRODUCTION}

Over the last three decades the FE method has firmly established itself as the standard approach for problems in computational solid mechanics (CSM), especially with regard to deformation problems involving non-linear material analysis [1,2]. As a contemporary, the FV method has similarly established itself within the field of computational fluid dynamics (CFD) [3, 4]. Both classes of methods integrate governing equations over pre-defined control volumes [3, 5], which are associated with the elements making up the domain of interest. Additionally, both approaches can be classified as weighted residual methods where they differ with respect to the weighting functions that are adopted [6].

Over the last decade a number of researchers have applied FV methods to problems in CSM (see [7] for a review) and it is now possible to classify these methods into two approaches, cell-centred $[8,9,10,11,12]$ and vertex-based $[13,6,14,7]$.

The first approach is based upon traditional FV methods [3] that have been widely applied in in the context of CFD [4]. Subsequently, in the last decade such techniques have been applied to CSM problems involving structured [8,9] and unstructured meshes [15, 10, 11, 12]. With regard to these techniques, it should be noted that when solid bodies undergo deformation the application of mechanical boundary conditions is best affected if they can be set at the physical boundary. However, if the disretisation approach is cell-centred then displacements at the boundary, for example, have to be projected from the nearest node of discretisation. Therefore, cell-centred approximations may be problematic when considering complex geometries where displacements at the boundary are not prescribed and are determined as part of the simulation.

The second approach is based on traditional FE methods [2] and employs shape functions to describe the variation of an independent variable, such as displacement, over an element and is therefore well suited to complex geometries $[13,6,14]$. In a more general sense the approach can be classified as a 
cell-vertex FV method $[4,6]$. However, it should be noted that the approach presented in this paper is a specific class of cell-vertex methods that employs non-overlapping control volumes [16, 17, 18, 7]. For this reason the approach will be subsequently referred to as a vertex-based FV method to distinguish it from other cell-vertex techniques. Additionally, it is important to note that the approach is equivalent to the previous non-overlapping FV methods as employed by Bailey and Cross [14] for 3D linear elastic problems and by Oñate et al. for 2D linear elastic problems [6].

Both the above FV approaches apply strict conservation over a control volume and have demonstrated superiority over traditional FE methods with regard to accuracy [10, 7]. Some researchers have attributed this to the local conservation of an independent variable as enforced by the control volumes employed $[13,14]$ and others to the enforced continuity of the derivatives of the independent variables across cell boundaries [10]. The objectives of this paper are to describe the application of a vertex-based FV method to problems involving elasto-plastic deformation, to describe implementations and to provide a detailed comparison with a standard Galerkin FE method for an extended range of 3D elements, consisting of tetrahedral, pentahedral and hexahedral types.

\section{MATHEMATICAL FORMULATION}

In this section standard mathematical models that have been employed generally in computational solid mechanics are presented. The models are described in a general sense with regard to dimensionality, such that formulations in any dimension are possible when suitable matrices and vectors are employed [7]. 


\subsection{Equilibrium Equations and Boundary Conditions}

In matrix form, the incremental equilibrium equations are

$$
[L]^{T}\{\Delta \sigma\}+\{b\}=\{0\} \quad \text { in } \Omega
$$

where $[L]$ is the differential operator, $\{\Delta \sigma\}$ is the Cauchy stress, $\{b\}$ is the body force and $\Omega$ is is the domain. The boundary conditions on the surface $\Gamma=\Gamma_{t} \cup \Gamma_{u}$ of the domain $\Omega$ can be defined as $[2,6]$

$$
\begin{aligned}
{[R]^{T}\{\Delta \sigma\} } & =\left\{t_{p}\right\} & \text { on } \Gamma_{t} \text { and } \\
\{\Delta u\} & =\left\{u_{p}\right\} & \text { on } \Gamma_{u},
\end{aligned}
$$

where $\left\{t_{p}\right\}$ are the prescribed tractions on the boundary $\Gamma_{t},\left\{u_{p}\right\}$ are the prescribed displacements on the boundary $\Gamma_{u}$ and $[R]$ is the outward normal operator $[6,7]$.

\subsection{Constitutive Relationship}

In matrix form, the stress is related to the elastic strain incrementally as follows; $\{\Delta \sigma\}=[D]\left\{\Delta \epsilon_{e}\right\}$, where $[D]$ is the elasticity matrix. For the deformation of metals, the von-Mises yield criterion is employed and the elastic strain is given by $\left\{\Delta \epsilon_{e}\right\}=\{\Delta \epsilon\}-\left\{\Delta \epsilon_{\mathrm{vp}}\right\}$, where $\{\Delta \epsilon\}$ and $\left\{\Delta \epsilon_{\mathrm{vp}}\right\}$ are the total and visco-plastic strain, respectively. The visco-plastic strain rate is given by the Perzyna model [19]

$$
\frac{d}{d t}\left\{\epsilon_{\mathrm{vp}}\right\}=\gamma\left\langle\frac{\sigma_{e q}}{\sigma_{y}}-1\right\rangle^{\frac{1}{N}} \frac{3}{2 \sigma_{e q}}\{s\},
$$

where $\sigma_{e q}, \sigma_{y}, \gamma, N$ and $s$ are the equivalent stress, yield stress, fluidity, strain rate sensitivity parameter and deviatoric stress, respectively. The $\langle x\rangle$ operator is defined as follows;

$$
<x>=\left\{\begin{array}{lll}
0 & \text { when } \quad x \leq 0 \quad \text { and } \\
x & \text { when } \quad x>0 .
\end{array}\right.
$$

The total infinitesimal strain is $\{\Delta \epsilon\}=[L]\{\Delta u\}$, where $\{\Delta u\}$ is the incremental displacement. 


\section{VERTEX-BASED DISCRETISATION}

Employing the constitutive relationship of the previous section in equations (1) and (2), and assuming the boundary conditions as described by equation (3) are directly satisfied by the vector $\{\Delta u\}$, the method of weighted residuals can be applied to the equations to obtain the following weak form of the equilibrium equation [2];

$$
\begin{gathered}
-\int_{\Omega}[L W]^{T}\left([D][L]\{\Delta u\}-[D]\left\{\Delta \epsilon_{\mathrm{vp}}\right\}\right) \mathrm{d} \Omega+\int_{\Omega}[W]^{T}\{b\} \mathrm{d} \Omega+ \\
\int_{\Gamma_{u}}[R W]^{T}\left([D][L]\{\Delta u\}-[D]\left\{\Delta \epsilon_{\mathrm{vp}}\right\}\right) \mathrm{d} \Gamma+\int_{\Gamma_{t}}[W]^{T}\left\{t_{p}\right\} \mathrm{d} \Gamma=\{0\}
\end{gathered}
$$

where $[W]$ is a diagonal matrix of arbitrary weighting functions.

At this point the unknown displacement can be approximated as [2]

$$
\{\Delta u\} \simeq\{\Delta \hat{u}\}=\sum_{j=1}^{n}[N]_{j}\{\Delta \bar{u}\}_{j}=\sum_{j=1}^{n}[I] N_{j}\{\Delta \bar{u}\}_{j}
$$

where $\{\Delta \bar{u}\}_{j}$ is the unknown displacement at the vertex $j, N_{j}$ is the shape function associated with the vertex and $[I]$ is the identity matrix. The displacement approximation can be introduced into equation (5) if the arbitrary weighting functions $[W]$ are replaced by a finite set of prescribed functions $[W]=\sum_{i=1}^{n}[W]_{i}$, for each vertex $i[2,6]$,

$$
\begin{gathered}
-\int_{\Omega}[L W]_{i}^{T}\left([D][L]\{\Delta \hat{u}\}-[D]\left\{\Delta \epsilon_{\mathrm{vp}}\right\}\right) \mathrm{d} \Omega+\int_{\Omega}[W]_{i}^{T}\{b\} \mathrm{d} \Omega+ \\
\int_{\Gamma_{u}}[R W]_{i}^{T}\left([D][L]\{\Delta \hat{u}\}-[D]\left\{\Delta \epsilon_{\mathrm{vp}}\right\}\right) \mathrm{d} \Gamma+\int_{\Gamma_{t}}[W]_{i}^{T}\left\{t_{p}\right\} \mathrm{d} \Gamma=\{0\} \\
\text { for } i=1, n .
\end{gathered}
$$

Equation (7) can be expressed as an incremental linear system of equations of the form $[K]\{\Delta \bar{u}\}-$ $\{f\}=\{0\}$, where $[K]$ is the global stiffness matrix, $\{\Delta \bar{u}\}$ is the global displacement approximation and $\{f\}$ is the global equivalent force vector and can be formed from the summation of the following Copyright (c) 2000 John Wiley \& Sons, Ltd. Int. J. Numer. Meth. Engng 2000; 00:1-41 Prepared using nmeauth.cls 
contributions;

$$
\begin{aligned}
{[K]_{i j}=} & \int_{\Omega_{i}}[L W]_{i}^{T}[D][L N]_{j} \mathrm{~d} \Omega-\int_{\Gamma_{u_{i}}}[R W]_{i}^{T}[D][L N]_{j} \mathrm{~d} \Gamma \quad \text { and } \\
\{f\}_{i}= & \int_{\Omega_{i}}[W]_{i}^{T}\{b\} \mathrm{d} \Omega+\int_{\Omega_{i}}[L W]_{i}^{T}[D]\left\{\Delta \epsilon_{\mathrm{vp}}\right\} \mathrm{d} \Omega \\
& +\int_{\Gamma_{t_{i}}}[W]_{i}^{T}\left\{t_{p}\right\} \mathrm{d} \Gamma-\int_{\Gamma_{u_{i}}}[R W]_{i}^{T}[D]\left\{\Delta \epsilon_{\mathrm{vp}}\right\} \mathrm{d} \Gamma
\end{aligned}
$$

where $\Omega_{i}$ is the control volume associated with the vertex $i$ and $\Gamma_{i}=\Gamma_{u_{i}} \cup \Gamma_{t_{i}}$ is the boundary of the control volume.

\subsection{Standard Galerkin FE Method}

In the standard Galerkin FE method the weighting function associated with a vertex is equal to the shape function of the unknown associated with that vertex $[2,4,6],[W]_{i}=[N]_{i}$. The shape functions describe the variation of an unknown over an element and there can be a number of elements associated with each vertex. Hence, it is apparent that control volumes described by weighting functions of this form will always overlap. This is illustrated in Figure 1(a) for a simple two dimensional case of two adjacent nodes $i$ and $j$, where the control volumes $\Omega_{i}$ and $\Omega_{j}$ have contributions from all the elements associated with their respective vertices $i$ and $j$.

Hence, for the standard Galerkin FE method the contributions as described by equations (8) and (9) are

$$
\begin{aligned}
{[K]_{i j} } & =\int_{\Omega_{i}}[B]_{i}^{T}[D][B]_{j} \mathrm{~d} \Omega \text { and } \\
\{f\}_{i} & =\int_{\Omega_{i}}[N]_{i}^{T}\{b\} \mathrm{d} \Omega+\int_{\Omega_{i}}[B]_{i}^{T}[D]\left\{\Delta \epsilon_{\mathrm{vp}}\right\} \mathrm{d} \Omega+\int_{\Gamma_{t_{i}}}[N]_{i}^{T}\left\{t_{p}\right\} \mathrm{d} \Gamma,
\end{aligned}
$$

where $[B]_{i}=[L N]_{i}$.

It is important to note that if the boundary of the control volume, such as that described by $\Gamma_{i}$ in Figure 1(a), coincides with the external boundary of the domain, the shape functions are not necessarily 
zero along that part of the boundary. Thus, if a flux is prescribed, such as a traction, this will not necessarily disappear and may contribute to the equivalent force vector as described in equation (11). Additionally, the symmetrical nature of the stiffness matrix as indicated by equation (10) should be noted. The Galerkin approach is accepted as the optimum technique for treating physical situations described by self-adjoint differential equations, particularly those in solid mechanics, as the inherent symmetrical nature is preserved by the choice of weighting functions $[2,6]$.

\subsection{Vertex-based FV Method}

In the vertex-based FV method the weighting functions associated with a vertex are equal to unity within the control volume, $[W]_{i}=[I]$, and zero elsewhere. This definition is equivalent to that for the subdomain collocation method as defined in the standard texts [4, 2]. However, it is important to note that weighting functions defined in this manner permit a variety of possibilities with regard to the control volume definition [6]. This is because the weighting functions are not restricted to a direct association with the cell or element as in the Galerkin case. This is an important consideration and requires the recognition of the vertex-based FV method as a discretisation technique in its own right [4].

For the vertex-based FV method the contributions as described by equations (8) and (9) are

$$
\begin{aligned}
{[K]_{i j} } & =-\int_{\Gamma_{u_{i}}}[R]_{i}^{T}[D][B]_{j} \mathrm{~d} \Gamma \quad \text { and } \\
\{f\}_{i} & =\int_{\Omega_{i}}\{b\} \mathrm{d} \Omega-\int_{\Gamma_{u_{i}}}[R]_{i}^{T}[D]\left\{\Delta \epsilon_{\mathrm{vp}}\right\} \mathrm{d} \Gamma+\int_{\Gamma_{t_{i}}}\left\{t_{p}\right\} \mathrm{d} \Gamma .
\end{aligned}
$$

It is important to note that the traction boundary conditions can be applied directly as another surface integral, but in the previous Galerkin approach an additional surface element is generally included on the domain boundary. A non-overlapping control volume definition suitable for a vertex-based FV method is illustrated in two and three dimensions in Figures 1(b) and 2, respectively. The Figures 
illustrate the assembling of vertex-based control volumes from their related sub-control volumes [7]. Additionally, the asymmetric nature of the contributions to the overall stiffness matrix as described by equation (12) does not ensure that symmetry will always be preserved. For this reason FV methods were initially argued as being inferior, but in the light of recent research where different control volume definitions have been proposed, the extent of this inferiority has come into question $[6,5,14]$.

\section{DESCRIPTION OF 3D ELEMENT TYPES}

In this section implementations of three dimensional element types for the standard FE and vertexbased FV methods will be described and compared. As part of this research a range of three dimensional element types has been developed by extending trilinear hexahedral (TLH) to include linear tetrahedral (LT) and bilinear pentahedral (BLP) element types with regard to the vertex-based FV method. General two dimensional element types have been described previously for linear elastic $[13,6]$ and non-linear material problems [20, 7].

It is possible to theoretically analyse and compare the FV and FE method for the LT element, as it is with linear elements in one and two dimensions [7, 6]. Unfortunately, as with higher order bilinear quadrilateral elements in the two dimensional case, no simple theoretical comparison is available with regard to the higher order bilinear or trilinear elements in three dimensions, though the same arguments apply with regard to closer agreement of the two methods in the limit of a suitably refined mesh [7].

The three dimensional elements discussed in this section are illustrated in both global and local coordinates in Figures 4, 5 and 6 respectively. The shape functions and associated derivatives are described in the Appendix for each three dimensional element type, respectively. Standard coordinate transformation techniques are employed for both the FE and vertex-based FV methods. The techniques are described in more detail in the following sections. As for the two dimensional case, equivalent 
meshes can be used by both methods, though it is important to note that the stiffness matrix contributions are computed differently [7]. For the FV method the construction of the sub-control volumes is a relatively straight forward extension of the two dimensional approach [13, 21], except that in three dimensions the control volumes are defined by internal surfaces of the mesh element $[14,7]$. In this way it is possible to construct a control volume consisting of cubic sub-control volume contributions from elements associated with a vertex. This approach is illustrated in Figure 2 for the simple case of eight arbitrary elements contributing to a vertex-based control volume. The control volume consists of the eight cubic sub-control volumes. Each sub-control volume has three integration points associated with it, which are situated at the face centres. It should be noted that it is possible to utilise a numerical integration scheme involving a greater number of weighted integration points. Although this approach is relatively straight forward it has not been investigated in the research presented here, as it involves further comparison of the two methods for higher order numerical integration point schemes. This research is restricted to comparing equivalent lower order integration schemes for the two methods. Finally, it should be noted that the case works equally well for a vertex with $n$ associated elements, where $n$ may consist of a variety of element types, such as tetrahedra, wedges or bricks.

In summation, equivalent elements are employed in both the FV and FE simulations. Therefore, the discretisation order, which is of course dependent upon the order of the shape functions associated with the elements used [2], is identical for both the FE and FV analyses.

\subsection{Linear Tetrahedral (LT) Element}

Naturally, the nodal points are equivalently defined in the local coordinate system for both FE and

FV methods. This is necessary in order to be consistent with the shape functions. The LT element is 
illustrated in local coordinates in Figure 4(b).

Obviously, the coordinates for the FE Gauss points and the FV integration points are different. To illustrate this difference the six integration points for the FV method are illustrated in Figures 7(b) and 7(c), whereas the single Gauss point is illustrated in Figure 7(a). The weighting associated with the Gauss point is equivalent to the volume the tetrahedron occupies in the local coordinate system. For the FV method the six integration points coincide with the six internal surfaces required to construct the four cubic sub-control volumes associated with a LT element.

4.1.1. Theoretical Analysis of the LT Element It is possible to theoretically analyse and compare both methods for the LT element due to its simple linear nature, by extending the two dimensional elastic analysis of a linear triangular element [6] to the three dimensional non-linear material analysis of LT element [7].

Concentrating on the non-linear terms of equations (11) and (13) the external force contributions at a node $i$ are

$$
\{f\}_{i}^{\mathrm{FE}}=-\int_{\Omega_{i}}\{B\}_{i}^{T}\{D\}\left\{\Delta \epsilon_{\mathrm{vp}}\right\} d \Omega \quad \text { and } \quad\{f\}_{i}^{\mathrm{FV}}=+\int_{\Gamma_{i}}\{R\}_{i}^{T}\{D\}\left\{\Delta \epsilon_{\mathrm{vp}}\right\} d \Gamma
$$

for the FE and FV methods respectively. The theoretical equivalence of these two integrals with regard to a LT element can now be proven. Consider a cluster of LT elements surrounding the vertex $i$ in a similar fashion to that described in Figure 2. The $k$ th component of the external force vector due to visco-plastic strains for the FE method with contributions from $n_{e}$ elements is

$$
f_{i k}^{\mathrm{FE}}=-\sum_{e=1}^{n_{e}} \int_{\Omega_{e_{i}}} \frac{\partial N_{e_{i}}}{\partial x_{j}} D_{e} \Delta \epsilon_{e_{j k}}^{(\mathrm{vp})} \mathrm{d} \Omega=\sum_{e=1}^{n_{e}} D_{e} \Delta \epsilon_{e_{j k}}^{(\mathrm{vp})}\left(-\int_{\Omega_{e_{i}}} \frac{\partial N_{e_{i}}}{\partial x_{j}} \mathrm{~d} \Omega\right)
$$

at node $i$. Alternatively, for the FV method it is

$$
f_{i k}^{\mathrm{FV}}=\sum_{e=1}^{n_{e}} \int_{\Gamma_{e i}} n_{j} D_{e} \Delta \epsilon_{e_{j k}}^{(\mathrm{vp})} \mathrm{d} \Gamma=\sum_{e=1}^{n_{e}} D_{e} \Delta \epsilon_{e_{j k}}^{(\mathrm{vp})}\left(\int_{\Gamma_{e_{i}}} n_{j} \mathrm{~d} \Gamma\right)
$$


In both cases the visco-plastic strain tensor is constant over the element, thus allowing the visco-plastic strain factor to be taken outside of the integral. This is a consequence of the linear nature of the element which furnishes strain and other associated constitutive variables as constants over the element. Hence, the contributions for the two methods are identical if the bracketed integrals in equations (14) and (15) are equivalent. In the three dimensional case it is possible to consider a single LT element from the cluster with surfaces of area $s_{1}, s_{2}, s_{3}$ and $s_{4}$ and unit outward normals $\left\{n_{1}\right\},\left\{n_{2}\right\},\left\{n_{3}\right\}$ and $\left\{n_{4}\right\}$. It should be noted that the LT element is assumed to be orientated such that surface $s_{4}$ is opposite vertex $i$.

Applying the divergence theorem [22] to the bracketed integral in equation (14), such that

$$
\int_{\Omega_{e_{i}}} \frac{\partial N_{e_{i}}}{\partial x_{j}} \mathrm{~d} \Omega=\int_{\partial \Omega_{e_{i}}} n_{j} N_{i} \mathrm{~d} \Gamma
$$

where $\partial \Omega_{e_{i}}$ is the boundary surface of the element. It can be shown analytically [7] that the integral of the linear shape functions associated with vertex $i$ over the boundary surface

$$
\int_{\partial \Omega_{e_{i}}} n_{j} N_{i} \mathrm{~d} \Gamma=n_{1_{j}} \frac{1}{3} s_{1}+n_{2_{j}} \frac{1}{3} s_{2}+n_{3_{j}} \frac{1}{3} s_{3}
$$

and by corollary of the divergence theorem

$$
n_{1_{j}} \frac{1}{3} s_{1}+n_{2_{j}} \frac{1}{3} s_{2}+n_{3_{j}} \frac{1}{3} s_{3}=-\int_{\Gamma_{e_{i}}} n_{j} \mathrm{~d} \Gamma,
$$

where $\Gamma_{e_{i}}$ is the elemental contribution to the $\mathrm{FV}$ control volume. By similar analytical procedures, it is also possible to demonstrate the equivalence of all contributions to the global system of equations for LT elements with regard to FE and FV methods.

\subsection{Bilinear Pentahedral (BLP) Elements}

The BLP element is described in local coordinates in Figure 5(b). The nine integration points for the FV method are drawn in three planes, Figures 9(a), 9(b) and 9(c), whereas the six Gauss points 
associated with the FE method are drawn in two planes in Figures 8(a) and 8(b). For the FV method the nine integration points coincide with the nine internal faces required to construct the six cubic subcontrol volumes associated with a BLP element. The elemental stiffness matrices formed from a BLP element are distinctive for the two methods. Additionally, for the FV method an asymmetric elemental contribution is added to the coefficient matrix for BLP elements when the sub-control volumes are not of equal volume, whereas for the FE method the contributions are again always symmetric regardless of element shape.

\subsection{Trilinear Hexahedral (TLH) Elements}

The TLH element is described in the local coordinate system in Figure 6(b). The twelve integration points for the FV method are drawn in three planes, Figures 11(a), 11(b) and 11(c), where as the eight Gauss points are drawn in two planes, Figures 10(a) and 10(b). For the FV method the twelve integration points coincide with the twelve internal surfaces required to construct the eight cubic subcontrol volumes associated with a TLH element. The elemental stiffness matrices formed from a TLH element are again different for the two methods. Additionally, for the FV method an asymmetric contribution to the coefficient matrix is obtained when the sub-control volumes are not of equal volume, whereas for the FE method the contributions are always symmetric regardless of the shape of the TLH element.

\section{RESULTS AND DISCUSSION}

In this section the vertex-based FV method is applied to two and three dimensional validation problems and compared with the standard Galerkin FE method. The non-linear solution procedure adopted for both methods is based upon that of Zienkiewicz and Cormeau $[23,7]$. Both methods utilised 
an explicit technique with regard to time stepping of the Perzyna equation (4). It is important to note that the FV solution procedure only differs from that of the FE in contributions to the global equivalent force vector and the global stiffness matrix, which allows an accurate comparison of the two methods [7]. Furthermore, the application of mechanical boundary conditions with regard to vertex-based values is identical for both procedures. However, appropriately weighted FV and FE formulations are employed with regard to the application of pressure and traction loads $[14,7] .\left(\_\right)$The methods are compared with regard to accuracy and computational cost. They are also analysed for a variety of meshes with different element assemblies.

\subsection{Test case: Perforated tensile strip}

The perforated tensile strip with linear strain hardening has been modelled extensively using traditional FE methods [23, 24] and a reference solution based upon experimental data is available [25]. The problem involves an applied stress as illustrated in Figure 12, which is increased incrementally. The initial increment loads the strip to the yield point and the following load increments cause plastic deformation up to the point of plastic flow. The six load increments are described in Table I. The material under investigation was an aluminium alloy, the Youngs modulus and Poisson ratio required to define the elasticity matrix are $7,000 \mathrm{~kg} \mathrm{~mm}^{-2}$ and 0.2 , respectively, the yield stress is $24.3 \mathrm{~kg} \mathrm{~mm}^{-2}$ and the linear strain hardening coefficient [1] is $232.5 \mathrm{~kg} \mathrm{~mm}^{-2}$. The material property values and units are consistent with those employed in both the original experimental [25] and numerical [23] analyses.

The total strain was measured using a birefringent coating technique on the perforated tensile strip [25]. The total strain profiles obtained along the minimum section of the perforated tensile strip, which is the line $\mathrm{X}-\mathrm{X}^{\prime}$ in Figure 12, are described for all load increments in Figure $13^{3}$. 
The perforated tensile strip can be modelled using a plane stress approximation, as described in Figure 12. The geometry of this problem requires a non-orthogonal mesh with regard to BLQ elements as also illustrated in Figure 12. The problem can also be modelled in two dimensions using CST elements. The FV and the FE methods are compared for meshes consisting of BLQ and CST elements.

The elasto-visco-plastic solution of this problem is time independent and the steady state solution is equivalent to the solution obtained in an elasto-plastic analysis [23]. An elasto-plastic numerical analysis with a von-Mises yield criterion has been performed using the commercial engineering software ANSYS [26], in order to provide a further reference solution. An identical mesh, using BLQ elements as described in Figure 12, was employed. The total strain profile obtained is described in Figure $13^{4}$.

It is important to note that previous FE analyses have largely over predicted the strain values when compared to the reference solution [23]. The same over prediction occurs in the numerical analyses performed in this research using both FE and FV methods, as illustrated in Figures $13^{1}$ and $13^{2}$ respectively. The problem was modelled with a number of meshes consisting of BLQ and CST elements, with varying mesh density [7]. The mesh density is summarised in Figure 12 for BLQ elements. The CST mesh employs the same number of nodes, but uses 630 elements. These meshes were fine enough to ensure that the numerical results are mesh independent [7]. Confirming the above theoretical analysis, the results for both methods are in complete agreement when CST elements are employed as illustrated in Figure 14(b). For BLQ elements, the two methods are generally in close agreement, but it is interesting to note that they are in closest agreement when the problem is loaded initially than at the the final load increment VI, as illustrated in Figure 14(a). At the final load increment the tensile piece is undergoing total strains of several percent, and the infinitesimal strain theory is reaching the limit of applicability. At this stage plastic flow is beginning to occur and the material non- 
linearity would begin to be augmented by geometrical non-linearity. Interestingly, the two methods appear to differ more as the overall non-linearity of the problem increases.

For this validation problem, the non-uniform structure of the mesh with regard to BLQ elements requires a bi-conjugate gradient method (BiCGM) for the solution of the asymmetric coefficient matrix assembled by the FV method, where as the symmetric coefficient matrix assembled by the FE method merely requires a conjugate gradient method (CGM). The computational expense of the BiCGM with regard to the comparison of the methods is illustrated in Figure 15(a), where the compute time is plotted against mesh density. As expected the FV is approximately twice as expensive as the FE method, because the BiCGM is computationally twice as expensive as the CGM. It should be noted that for comparison purposes it is possible to apply the BiCGM to both a symmetric and an asymmetric matrix. However, the application of the BiCGM to a symmetric matrix is computationally wasteful as it requires twice the computational cost to obtain an identical solution to the CGM. Therefore, such a comparison has not been performed in this paper. For meshes consisting of CST elements the coefficient matrices obtained by FE and FV methods are identical, hence the CGM can be employed in both cases. The computational costs of the methods are in closer agreement as illustrated in Figure 15(b). The FV is approximately ten percent slower than the FE method, this is attributable to the larger number of integration points associated with the FV method for CST elements [7].

\subsection{Test case: Internally pressurised spherical vessel}

For this validation problem a thick walled spherical vessel, consisting of an elastic-perfectly plastic material, undergoes an instantaneously applied internal pressure load. The pressure load is 30 $\mathrm{dN} \mathrm{mm}^{-2}$, the Youngs modulus and Poisson ratio required to define the elasticity matrix are 21,000 $\mathrm{dN} \mathrm{mm}{ }^{-2}$ and 0.3 , respectively, and the yield stress is $24 \mathrm{dN} \mathrm{mm}^{-2}$. The material property values 
and units are consistent with those employed in the plane strain analysis of a thick walled cylinder [1]. This problem is rate independent and the final solution is equivalent to that of an elasto-plastic analysis [23]. A closed form radial solution is available [27]. Numerically the problem can be modelled in three dimensional Cartesian coordinates, with the normal displacement components fixed to zero in the relative symmetry planes. The spherical vessel is then reduced to an octant as illustrated in Figure $3^{1}$.

Firstly, the problem was analysed with a series of meshes consisting of TLH elements [7]. The hoop stress profiles, along the radii, from a mesh independent solution are plotted and compared against the reference solution in Figure 16(a). The profiles illustrate the stress in the plastic and elastic regions, and the radial extent of the plastic region according to the analytical solution. The close agreement of the two methods is illustrated. However, it is important to note the closer agreement between the reference solution and the FV method when a coarse mesh is employed. These observations may be associated with the higher order, trilinear nature of the elements employed in the three dimensional analysis at this stage. With regard to the FV method, the implementation of pressure loads (tractions) will involve bilinear face elements for TLH elements. Hence, when considering the application of pressure loads for the two methods as described in equations (11) and (13), the contributions are different due to the individual weighting technique associated with each method. Furthermore, the weighting technique employed for the FV method may be more complementary, when applied generally, as all the terms are integrated conservatively at a local level. Conversely, for the FE method the weighting is not locally conservative which may introduce errors when pressure loads are employed. These conclusions are tentative and rely on the interpretation of the present observations, but they agree with the findings of other researchers [10] and strongly suggest that further research of the FV method is worthwhile. It should also be noted that a comparison of mesh independent solutions has been 
performed by implementing a strategy of mesh refinement and that the results shown in Figure 16 are mesh independent [7].

Secondly, the problem was analysed with a series of meshes consisting of BLP elements and there was much closer agreement between the methods [7]. This is attributable to the lower order, bilinear nature of the element concerned and the linear nature of the triangular faces over which the pressure loads were applied. As illustrated in Figure $3^{3}$ the BLP elements are orientated so the pressure load was prescribed over a triangular face. This was an outcome of the automatic mesh generator employed [28] and it is possible to further study the element when pressures are applied to the bilinear, quadrilateral faces.

Thirdly, the problem was analysed with a series of meshes consisting of LT elements [7]. The hoop stress profiles from mesh independent solutions are plotted in Figure 16(b). As expected, there is complete agreement between the two methods with regard to LT elements as the global stiffness matrices and global force vectors constructed by each method are theoretically identical, as shown earlier in the paper. This is a consequence of the linear nature of both the element concerned and the triangular faces over which the pressure is applied.

Finally, the methods were compared with regard to computational cost. Considering LT elements, as the matrices are identical and symmetric a CGM is applicable in both cases. As illustrated in Figure 17(b), the FV method (FV-CGM) requires more CPU time than the FE method (FE-CGM) even when the same linear solver is employed. This is expected as the FV method visits six integration points, while the FE method visits a single Gauss point when adding contributions to the linear system of equations [7].

Considering TLH elements, the geometrical nature of this validation problem prohibits an orthogonally assembled mesh. Hence, for the FV method a BiCGM is required due to the asymmetric 
nature of the coefficient matrix obtained [7]. Conversely, for the FE method a CGM is sufficient as the matrix obtained is symmetric. These requirements agree with the discussions in the previous section. As illustrated in Figure 17(a), the FV method (FV-BiCGM) requires approximately twice the CPU time as the FE method (FE-CGM). This is also expected due to the computational requirements of the two different linear solvers employed. Also for TLH elements, the FV method visits twelve integration points per element, while the FE method visits eight Gauss points per element.

Hence, it can finally be concluded that any improvement in accuracy obtained by employing the vertex-based FV method must be offset against the extra computational cost required.

\section{CONCLUSIONS}

The aim of the research presented in this paper was to develop and investigate an elesto-visco-plastic procedure that can fit within a three-dimensional FV multi-physics simulation framework. From the investigation of the procedure it emerges that:

- The vertex-based FV discretisation gives rise to an asymmetrical stiffness matrix for higher order elements in both 2D and 3D.

- For linear elements the stiffness matrix is symmetrical (as for the standard Galerkin FE method) and the solution times for both methods are reasonably close; the FV approach is approximately $10 \%$ more expensive than the otherwise equivalent FE method because of the larger number of integration points.

- For higher order elements the asymmetric system matrix, arising from the FV formulation, requires an appropriate solution method and in the case of the $\operatorname{BiCGM}(\underline{\operatorname{aBiCGM} \text { and }})$ this is twice as expensive as the symmetric CGM. 
- At the same level of mesh density and element type the vertex-based FV discretisation provides results that are very similar to those of standard Galerkin FE methods.

The ultimate objective of this research is to develop a framework of numerical procedures for solving a range of physical continuum phenomena in a compatible manner and to therefore facilitate the analysis of problems involving the closely coupled interactions of such phenomena (ie. multi-physics). The procedure developed in this research has been included in the multi-physics simulation software, PHYSICA [29], which has been applied to a range of problems involving non-linear material behaviour $[30,31,32,33]$.

ACKNOWLEDGEMENTS

The authors are grateful for the financial support of the EPRSC with regard to the funding of this research project.

\section{APPENDIX}

\section{LT Element}

Shape functions,

$$
\begin{aligned}
& N_{1}(s, t, u)=\quad \frac{1}{4}+\frac{2}{3} s-\frac{1}{3 \sqrt{2}} u, \quad N_{2}(s, t, u)=\frac{1}{4}-\frac{1}{3} s+\frac{2 \sqrt{3}}{6} t-\frac{1}{3 \sqrt{2}} u, \\
& N_{3}(s, t, u)=\frac{1}{4}-\frac{1}{3} s-\frac{2 \sqrt{3}}{6} t-\frac{1}{3 \sqrt{2}} u, \quad N_{4}(s, t, u)=\quad \frac{1}{4}+\frac{1}{\sqrt{2}} u .
\end{aligned}
$$

Derivatives,

$$
\begin{array}{rlrlrlrl}
\frac{\partial N_{1}}{\partial s} & = & \frac{2}{3}, & \frac{\partial N_{1}}{\partial t} & = & 0, & \frac{\partial N_{1}}{\partial u} & =-\frac{1}{3 \sqrt{2}}, \\
\frac{\partial N_{2}}{\partial s}=-\frac{1}{3}, & \frac{\partial N_{2}}{\partial t} & = & \frac{2 \sqrt{3}}{6}, & \frac{\partial N_{2}}{\partial u} & =-\frac{1}{3 \sqrt{2}}, \\
\frac{\partial N_{3}}{\partial s}=-\frac{1}{3}, & \frac{\partial N_{3}}{\partial t}= & -\frac{2 \sqrt{3}}{6}, & & \frac{\partial N_{3}}{\partial u}=-\frac{1}{3 \sqrt{2}}, \\
\frac{\partial N_{4}}{\partial s}= & 0, & \frac{\partial N_{4}}{\partial t} & = & 0, & \frac{\partial N_{4}}{\partial u} & =\frac{1}{\sqrt{2}} .
\end{array}
$$




\section{BLP Element}

Shape functions,

$$
\begin{array}{rrrrrr}
N_{1}(s, t, u) & = & \frac{1}{6}(1+2 s)(1-u), & N_{2}(s, t, u) & = & \frac{1}{6}(1-s+\sqrt{3} t)(1-u), \\
N_{3}(s, t, u)= & \frac{1}{6}(1-s-\sqrt{3} t)(1-u), & N_{4}(s, t, u) & & \frac{1}{6}(1+2 s)(1+u), \\
N_{5}(s, t, u)= & \frac{1}{6}(1-s+\sqrt{3} t)(1+u), & & N_{6}(s, t, u)= & \frac{1}{6}(1-s-\sqrt{3} t)(1+u) .
\end{array}
$$

Derivatives,

$$
\begin{aligned}
& \frac{\partial N_{1}}{\partial s}=\frac{1}{3}(1-u), \quad \frac{\partial N_{1}}{\partial t}=0, \quad \frac{\partial N_{1}}{\partial u}=\quad-\frac{1}{6}(1+2 s), \\
& \frac{\partial N_{2}}{\partial s}=-\frac{1}{6}(1-u), \quad \frac{\partial N_{2}}{\partial t}=\quad \frac{\sqrt{3}}{6}(1-u), \quad \frac{\partial N_{2}}{\partial u}=-\frac{1}{6}(1-s+\sqrt{3} t), \\
& \frac{\partial N_{3}}{\partial s}=-\frac{1}{6}(1-u), \quad \frac{\partial N_{3}}{\partial t}=-\frac{\sqrt{3}}{6}(1-u), \quad \frac{\partial N_{3}}{\partial u}=-\frac{1}{6}(1-s-\sqrt{3} t), \\
& \frac{\partial N_{4}}{\partial s}=\frac{1}{3}(1+u), \quad \frac{\partial N_{4}}{\partial t}=0, \quad \frac{\partial N_{4}}{\partial u}=\frac{1}{6}(1+2 s), \\
& \frac{\partial N_{5}}{\partial s}=-\frac{1}{6}(1+u), \quad \frac{\partial N_{5}}{\partial t}=\frac{\sqrt{3}}{6}(1+u), \quad \frac{\partial N_{5}}{\partial u}=\frac{1}{6}(1-s+\sqrt{3} t), \\
& \frac{\partial N_{6}}{\partial s}=-\frac{1}{6}(1+u), \quad \frac{\partial N_{6}}{\partial t}=-\frac{\sqrt{3}}{6}(1+u), \quad \frac{\partial N_{6}}{\partial u}=\frac{1}{6}(1-s-\sqrt{3} t),
\end{aligned}
$$

\section{TLH Element}

Shape functions,

$$
\begin{array}{lll}
N_{1}(s, t, u) & =\frac{1}{8}(1+s)(1+t)(1+u), & N_{2}(s, t, u)=\frac{1}{8}(1-s)(1+t)(1+u), \\
N_{3}(s, t, u)=\frac{1}{8}(1-s)(1-t)(1+u), & N_{4}(s, t, u)=\frac{1}{8}(1+s)(1-t)(1+u), \\
N_{5}(s, t, u)=\frac{1}{8}(1+s)(1+t)(1-u), & N_{6}(s, t, u)=\frac{1}{8}(1-s)(1+t)(1-u), \\
N_{7}(s, t, u)=\frac{1}{8}(1-s)(1-t)(1-u), & N_{8}(s, t, u)=\frac{1}{8}(1+s)(1-t)(1-u) .
\end{array}
$$


Derivatives,

$$
\begin{array}{rlrl}
\frac{\partial N_{1}}{\partial s}= & \frac{1}{8}(1+t)(1+u), & \frac{\partial N_{1}}{\partial t}= & \frac{1}{8}(1+s)(1+u), \\
\frac{\partial N_{2}}{\partial s}= & -\frac{1}{8}(1+t)(1+u), & \frac{\partial N_{2}}{\partial t}= & \frac{1}{8}(1-s)(1+u), \\
\frac{\partial N_{3}}{\partial s}= & -\frac{1}{8}(1-t)(1+u), & \frac{\partial N_{3}}{\partial t}= & -\frac{1}{8}(1-s)(1+u), \\
\frac{\partial N_{4}}{\partial s}= & \frac{1}{8}(1-t)(1+u), & \frac{\partial N_{4}}{\partial t}= & -\frac{1}{8}(1+s)(1+u), \\
\frac{\partial N_{5}}{\partial s}= & \frac{1}{8}(1+t)(1-u), & \frac{\partial N_{5}}{\partial t}= & \frac{1}{8}(1+s)(1-u), \\
\frac{\partial N_{6}}{\partial s}= & -\frac{1}{8}(1+t)(1-u), & \frac{\partial N_{6}}{\partial t}= & \frac{1}{8}(1-s)(1-u), \\
\frac{\partial N_{7}}{\partial s}= & -\frac{1}{8}(1-t)(1-u), & \frac{\partial N_{7}}{\partial t}= & -\frac{1}{8}(1-s)(1-u), \\
\frac{\partial N_{8}}{\partial s}= & \frac{1}{8}(1-t)(1-u), & \frac{\partial N_{8}}{\partial t}= & -\frac{1}{8}(1+s)(1-u), \\
\frac{\partial N_{1}}{\partial u} & = & \frac{1}{8}(1+s)(1+t), \\
\frac{\partial N_{2}}{\partial u} & = & \frac{1}{8}(1-s)(1+t), \\
\frac{\partial N_{3}}{\partial u} & = & \frac{1}{8}(1-s)(1-t), \\
\frac{\partial N_{4}}{\partial u} & = & \frac{1}{8}(1+s)(1-t), \\
\frac{\partial N_{5}}{\partial u} & = & -\frac{1}{8}(1+s)(1+t), \\
\frac{\partial N_{6}}{\partial u} & = & -\frac{1}{8}(1-s)(1+t), \\
\frac{\partial N_{7}}{\partial u} & = & -\frac{1}{8}(1-s)(1-t), \\
\frac{\partial N_{8}}{\partial u} & = & -\frac{1}{8}(1+s)(1-t) .
\end{array}
$$

\section{REFERENCES}

1. Owen DRJ and Hinton E. Finite Elements in Plasticity: Theory and Practice. Pineridge Press Ltd., Swansea, UK, 1980.

2. Zienkiewicz OC and Taylor RL. The Finite Element Method: Volume 1: Basic Formulation and Linear Problems. MagrawHill, Maidenhead, Berkshire, UK, 1989.

3. Patanker SV. Numerical Heat Transfer and Fluid Flow. Hemisphere, Washington DC, 1980.

4. Hirsch C. Numerical Computation of Internal and External Flows: Fundamentals of Numerical Discretisation, volume 1. John Wiley and Sons, 1988.

5. Zienkiewicz OC. Origins, milestones and directions of the finite element method - a personal view. Archives of computational methods in Engg. 1995; 2:1-48. 
6. Oñate E, Cervera M and Zienkiewicz OC. A finite volume format for structural mechanics. Int. Journal for Num. Methods in Engg. 1994; 37:181-201.

7. Taylor GA. A Vertex Based Discretisation Scheme Applied to Material Non-linearity within a Multi-physics Finite Volume Framework. PhD thesis, The University of Greenwich, 1996.

8. Demirdzic I and Martinovic D. Finite volume method for thermo-elasto-plastic stress analysis. Computer Methods in Applied Mechanics and Engineering 1992; 109:331-349.

9. Hattel JH and Hansen PN. A control volume-based finite difference method for solving the equilibrium equations in terms of displacements. Appl. Math. Modelling 1995; 19:210-243.

10. Wheel MA. A geometrically versatile finite volume formulation for plane elastostatic stress analysis. Journal of Strain Analysis 1996; 31(2):111-116.

11. Wheel MA. A mixed finite volume formulation for determining the small strain deformation of incompressible materials. Int. Journal for Num. Methods in Engg. 1999; 44:1843-1861.

12. Jasak H and Weller HG. Application of the finite volume method and unstructured meshes to linear elasticity. Int. Journal for Num. Methods in Engg. 2000; 48:267-287.

13. Fryer YD, Bailey C, Cross M and Lai C-H. A control volume procedure for solving the elastic stress-strain equations on an unstructured mesh. Appl. Math. Modelling 1991; 15:639-645.

14. Bailey $\mathrm{C}$ and Cross M. A finite volume procedure to solve elastic solid mechanics problems in three dimensions on an unstructured mesh. Int. Journal for Num. Methods in Engg. 1995; 38:1757-1776.

15. Demirdzic I and Muzaferija S. Finite volume method for stress analysis in complex domains. Int. Journal for Num. Methods in Engg. 1994; 37:3751-3766.

16. Baliga BR and Patanker SV. A new finite-element formulation for convection-diffusion problems. Numerical Heat Transfer $1980 ; 3: 393-409$.

17. Selmin V. The node-centred finite volume approach: Bridge between finite differences and finite elements. Computer Methods in Applied Mechanics and Engineering 1992; 102:107-138.

18. Idelsohn SR and Oñate E. Finite volumes and finite elements: Two 'good friends'. Int. Journal for Num. Methods in Engg. $1994 ; 37: 3323-3341$.

19. Perzyna P. Fundamental problems in visco-plasticity. Advan. Appl. Mech. 1966; 9:243-377.

20. Taylor GA, Bailey C and Cross M. Solution of the elastic/visco-plastic constitutive equations: A finite volume approach. Appl. Math. Modelling 1995; 19:746-760.

21. Fryer YD. A Control Volume Unstructured Grid Approach to the Solution of the Elastic Stress-Strain Equations. PhD thesis, The University of Greenwich, 1993. 
22. Bourne DE and Kendal PC. Vector Analysis and Cartesian Tensors. The Camelot Press Ltd., Southampton, UK, 1980.

23. Zienkiewicz OC and Cormeau IC. Visco-plasticity—plasticity and creep in elastic solids—a unified numerical solution approach. Int. Journal for Num. Methods in Engg. 1974; 8:821-845.

24. Zienkiewicz OC and Taylor RL. The Finite Element Method: Volume 2: Solid and Fluid Mechanics, Dynamics and Nonlinearity. Magraw-Hill, Maidenhead, Berkshire, UK, 1991.

25. Theocaris PS and Marketos E. Elastic-plastic analysis of perforated thin strips of a strain-hardening material. J. Mech. Phys. Solids 1964; 12:377-390.

26. Swanson Analysis Systems, Inc., Houston, USA. ANSYS V5.0.

27. Hill R. The Mathematical Theory of Plasticity. Clarendon Press, Oxford, UK, 1950.

28. Femview Ltd., Leicester, UK. FEMGEN/FEMVIEW.

29. MPS Ltd, London, UK. (http://www.multi-physics.com). PHYSICA.

30. Taylor GA, Bailey C, and Cross M. Material non-linearity within a finite volume framework for the simulation of a metal casting process. In Owen DRJ, Oñate E, and Hinton E, editors, Fourth international conference on computational plasticity: Fundamentals and Applications: Pt. 2, 1459-1470, 1995.

31. Cross M, Bailey C, Pericleous KA, Bounds SM, Moran GJ, Taylor GA and McManus K. Computational modelling of interacting phenomena in the shape casting process. In Thomas BG and Beckerman C, editors, Modeling of Casting, Welding and Advanced Solidification Processes VIII, 787-794, 1998.

32. Bailey C, Wheeler D and Cross M. An integrated modeling approach to solder joint formation. IEEE Transactions on Components and Packaging Technologies 1999; 22(4):497-502.

33. Hua Lu, Bailey C and Cross M. Reliability analysis of flip chip designs via computer simulation. Journal of Electronic Packaging 2000; 122:214-219. 


\begin{tabular}{rcccccc}
\hline & I & II & III & IV & V & VI \\
\hline Increment $\left(\mathrm{kg} \mathrm{mm}^{-2}\right)$ & 5.59 & 0.95 & 1.46 & 1.73 & 1.52 & 1.64 \\
Total $\left(\mathrm{kg} \mathrm{mm}^{-2}\right)$ & 5.59 & 6.54 & 8.00 & 9.73 & 11.25 & 12.89 \\
\hline
\end{tabular}

Table I. Load increments applied to the perforated tensile strip. 


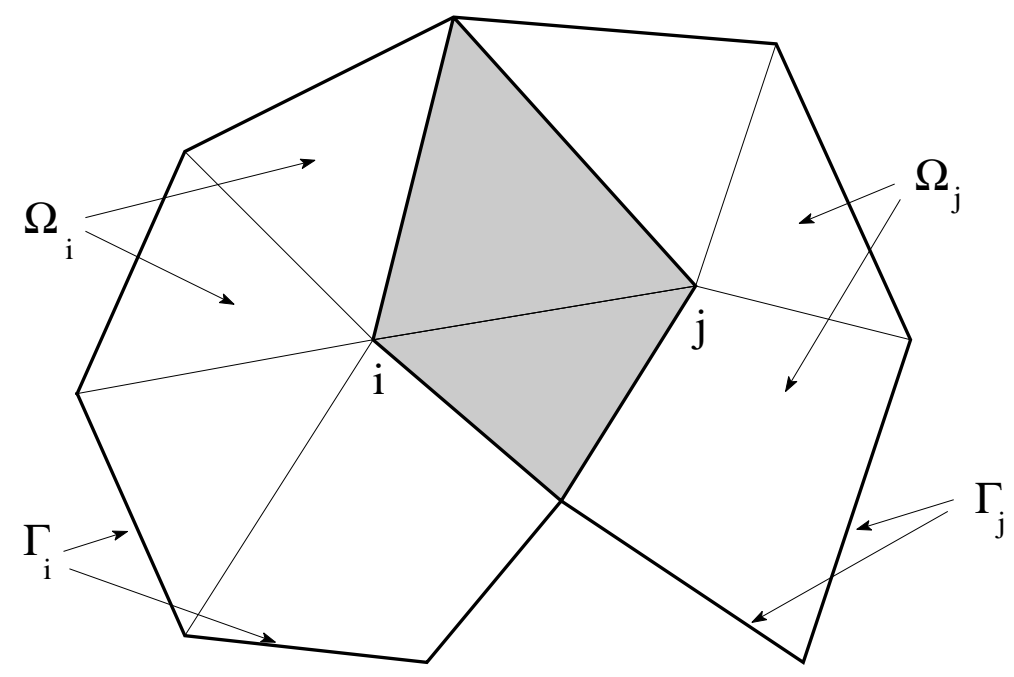

(a)

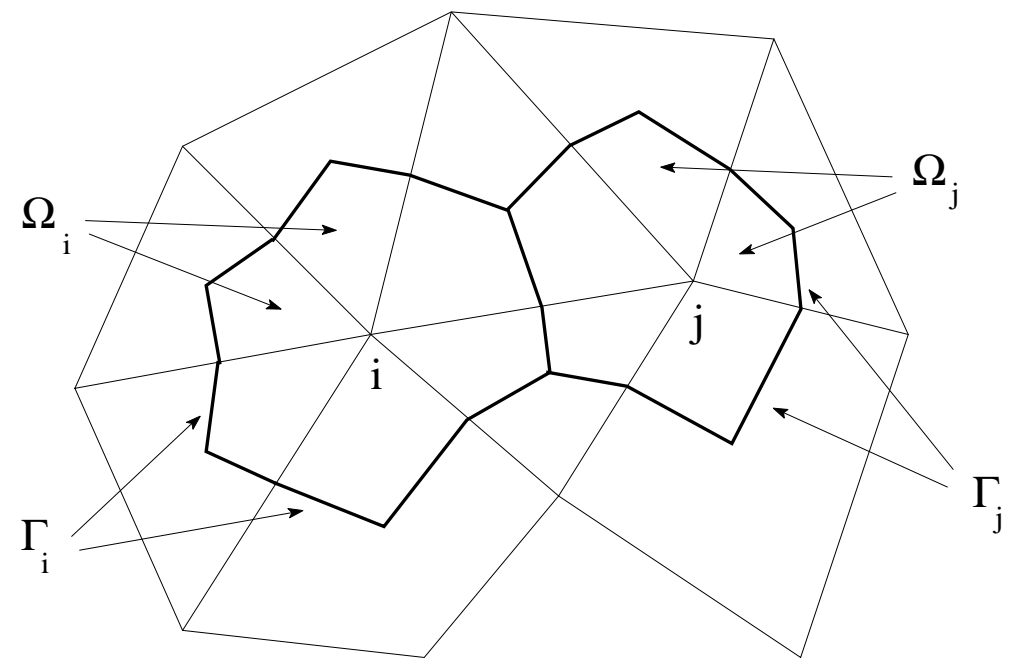

(b)

Figure 1. 2D control volumes, (a) overlapping FE and (b) non-overlapping FV. 

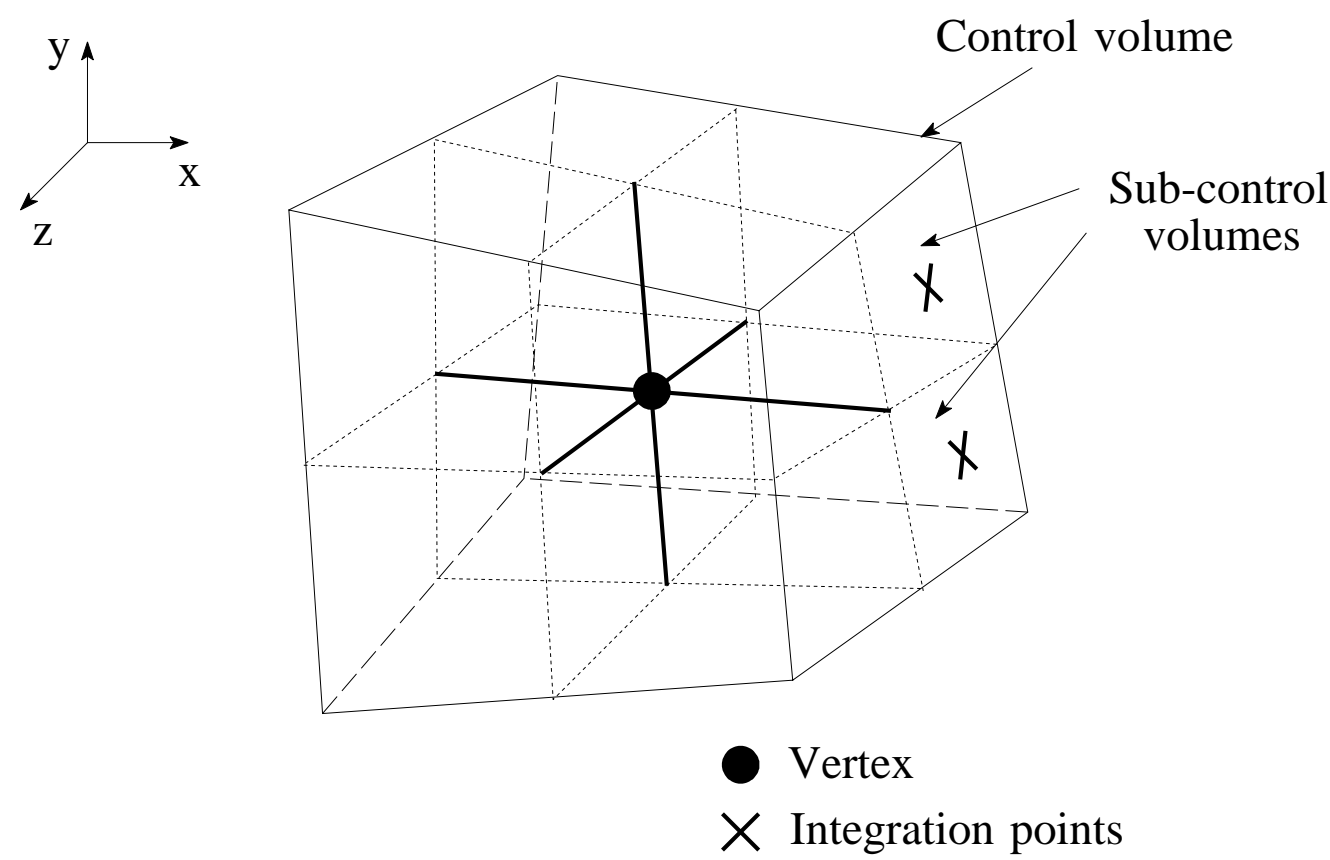

Figure 2. 3D assembly of FV sub - control volumes at a vertex. 

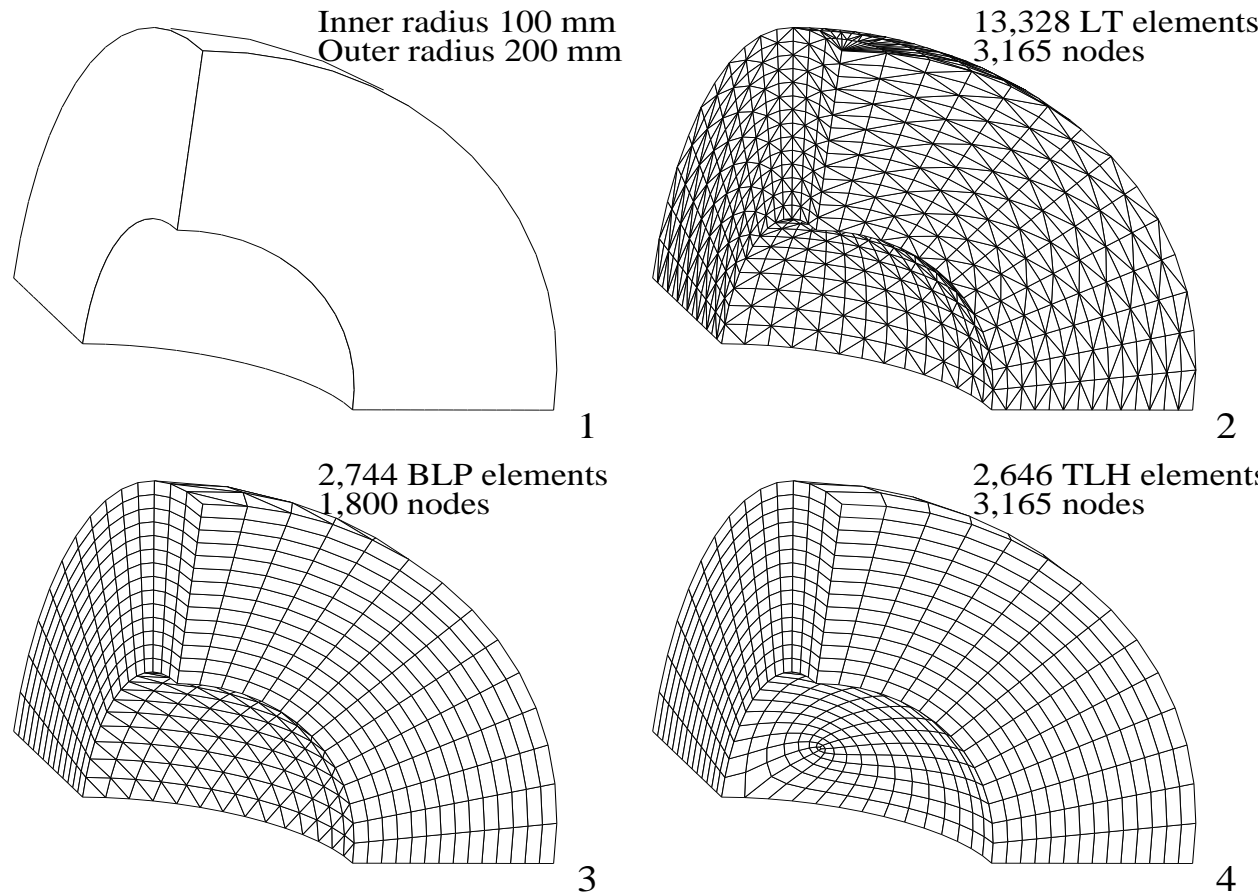

Figure 3. 3D schematic and unstructured meshes for a spherical vessel. 


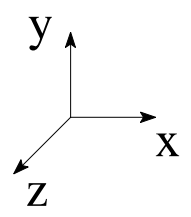

(a)
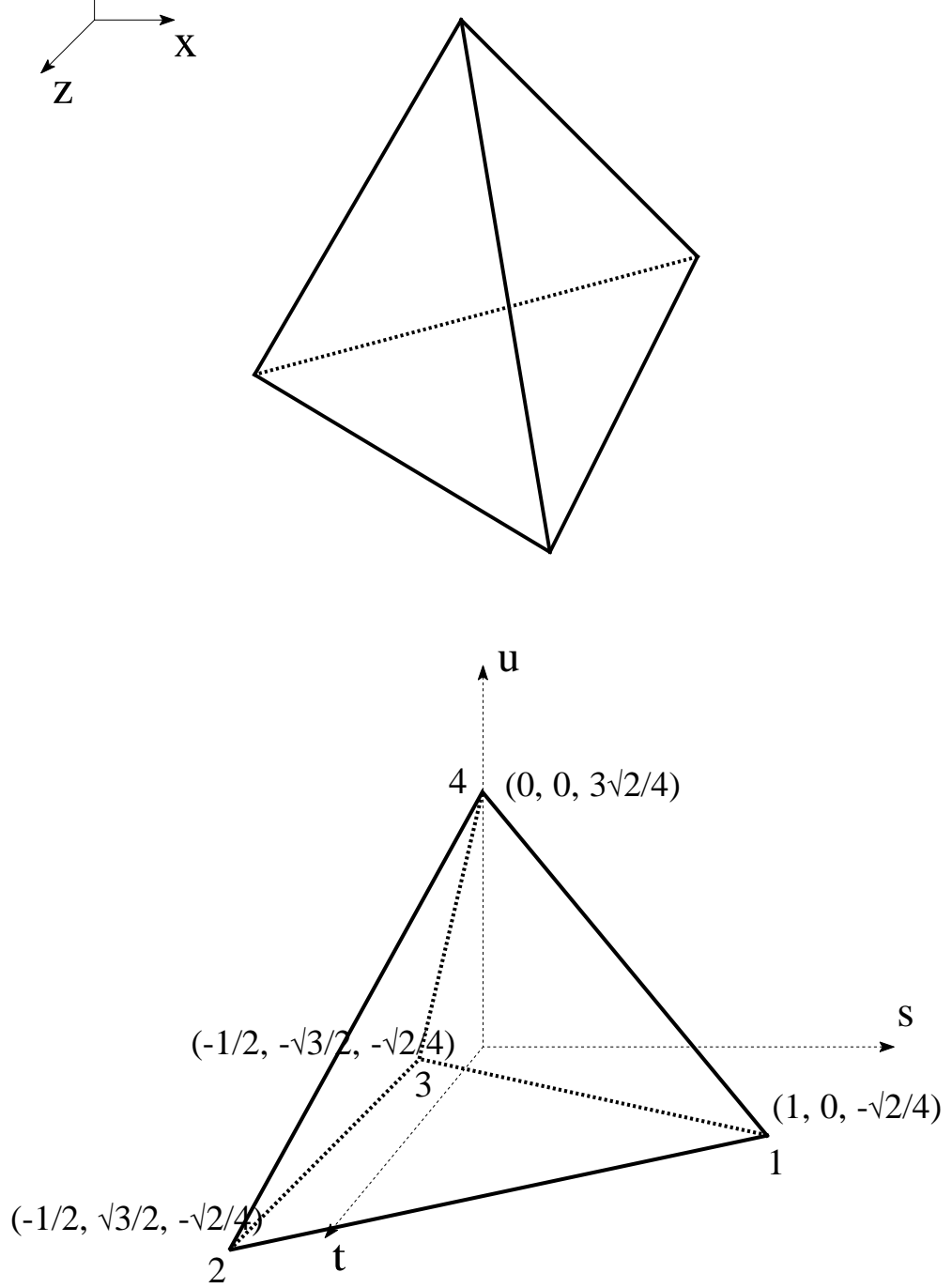

(b)

Figure 4. LT element in (a) global coordinates and (b) local coordinates. 


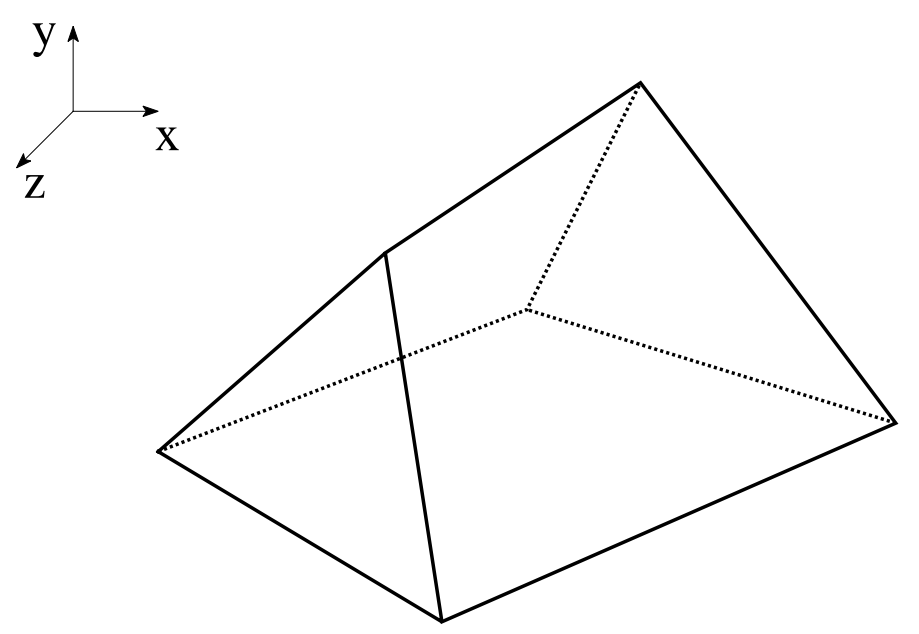

(a)

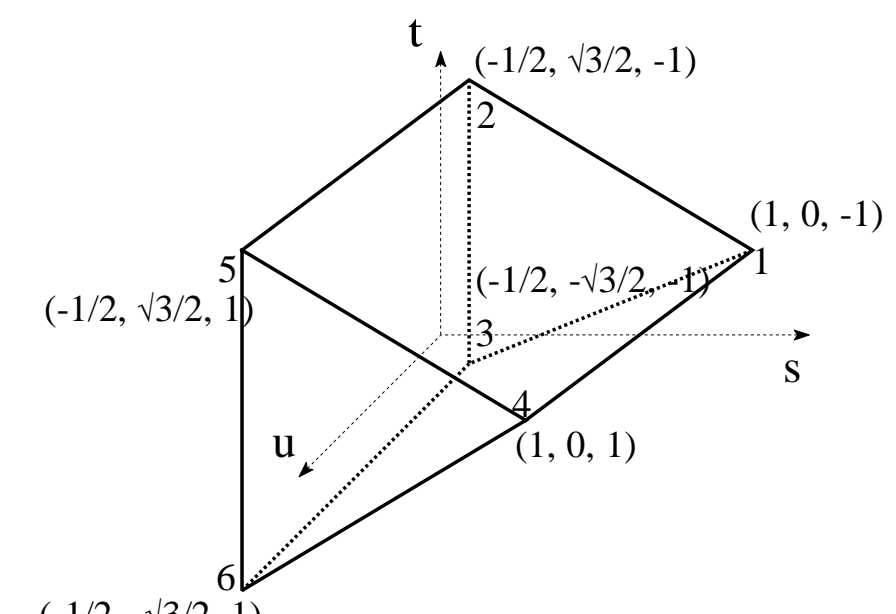

(b)

Figure 5. BLP element in (a) global coordinates and (b) local coordinates. 


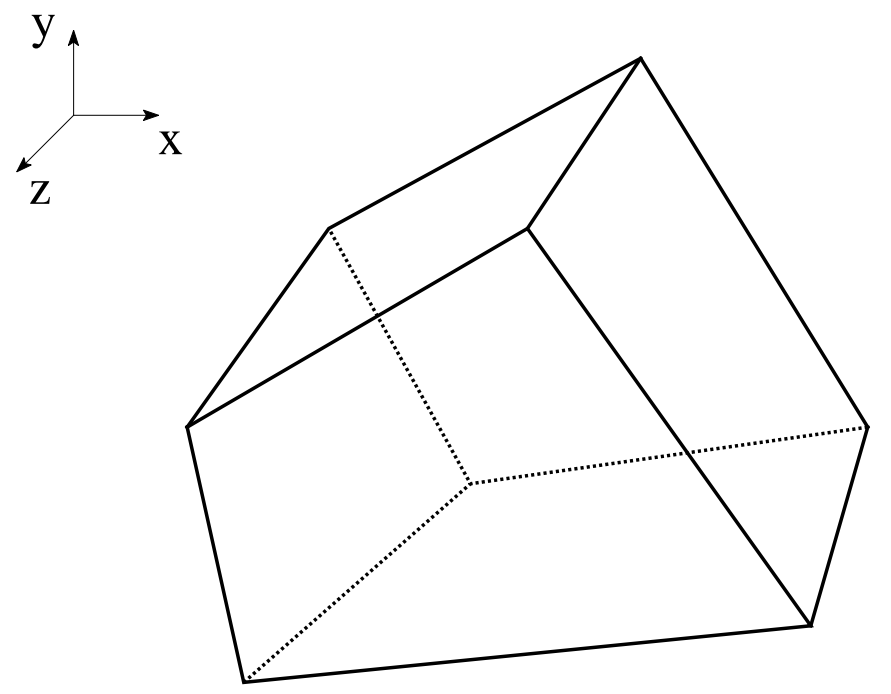

(a)

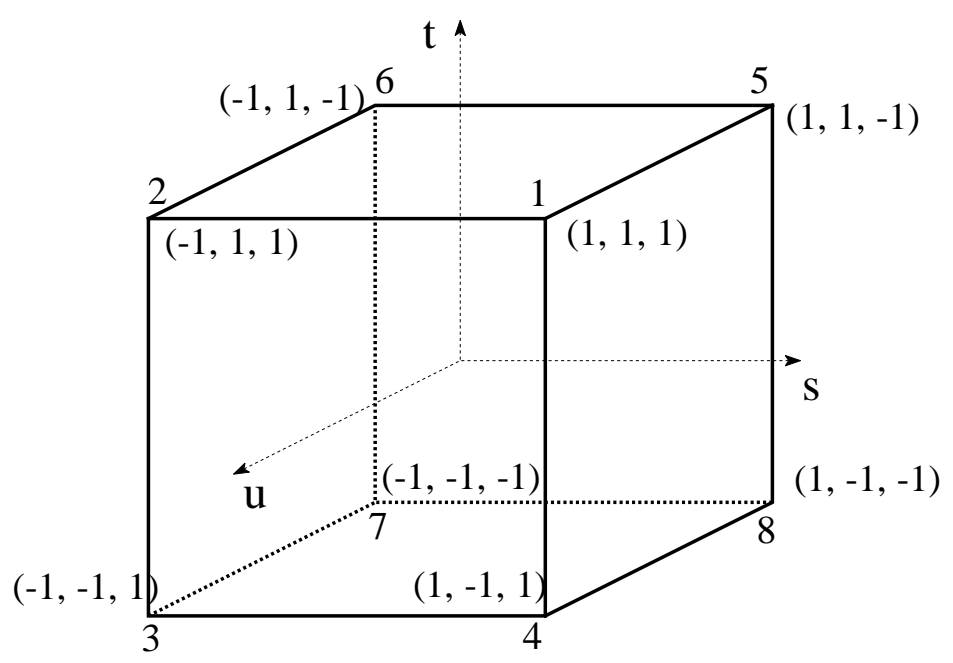

(b)

Figure 6. TLH element in (a) global coordinates and (b) local coordinates. 


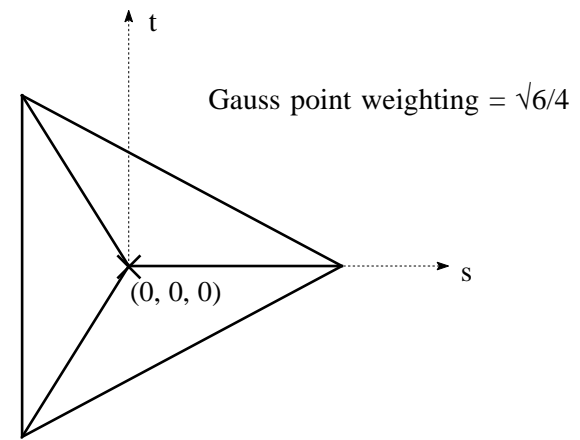

(a)

$$
X \text { Gauss points }
$$

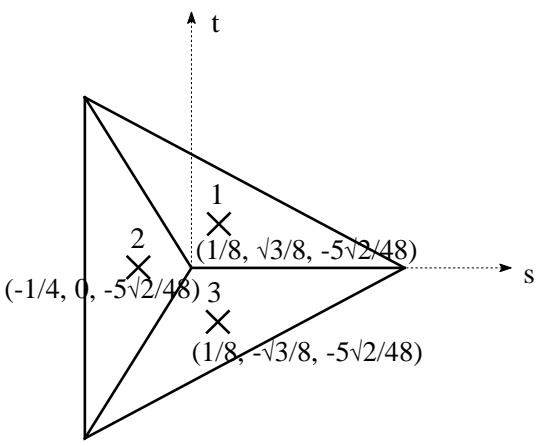

(b)

$$
X \text { Integration points }
$$

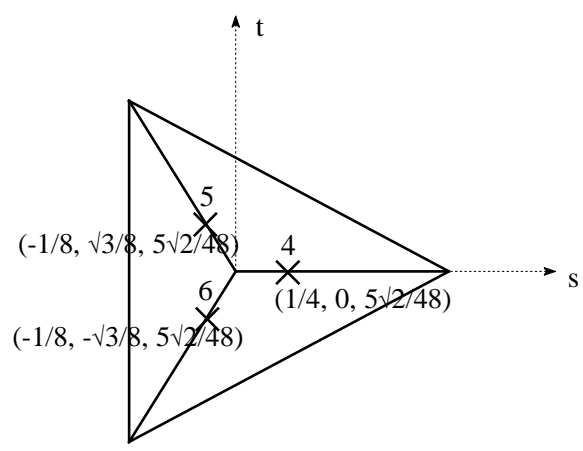

(c)

$$
X \text { Integration points }
$$

Figure 7. LT element in local coordinates, (a) Gauss point and, (b) vertically and (c) horizontally aligned( $\underline{\text { inclined }}$ ) integration points. 


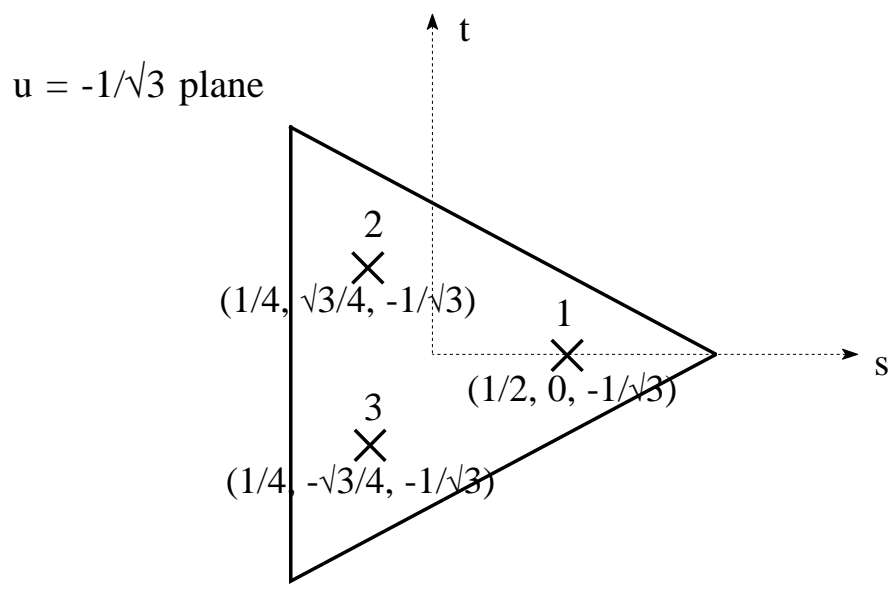

(a)

$$
X \text { Gauss points }
$$

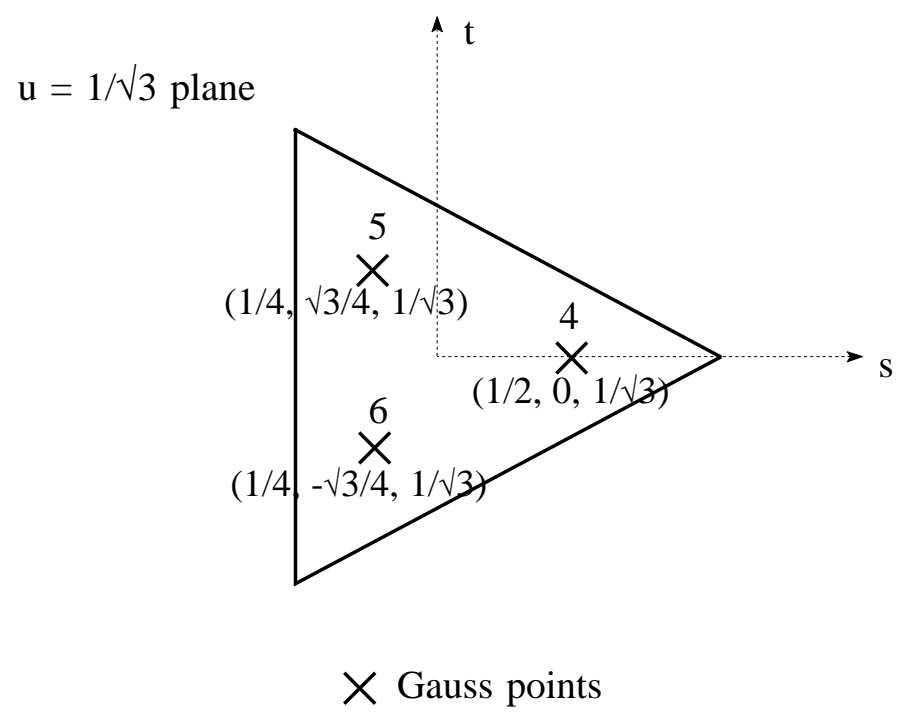

(b)

Figure 8. BLP Gauss points in local coordinates. (a) $u=-1 / \sqrt{ } 3$ and (b) $u=1 / \sqrt{ } 3$. 


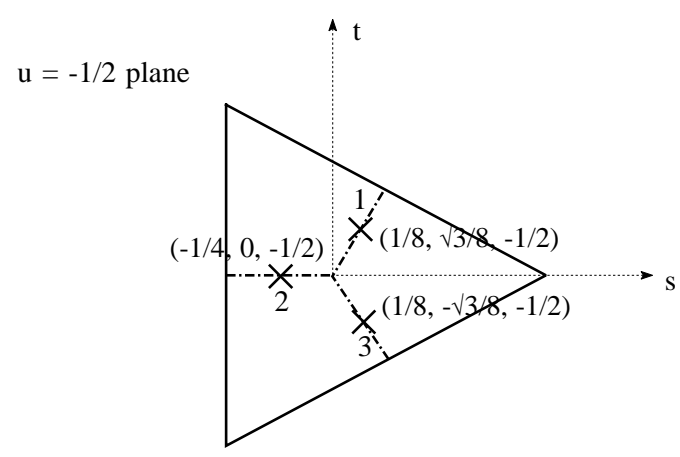

(a)

$$
\times \text { Integration points }
$$

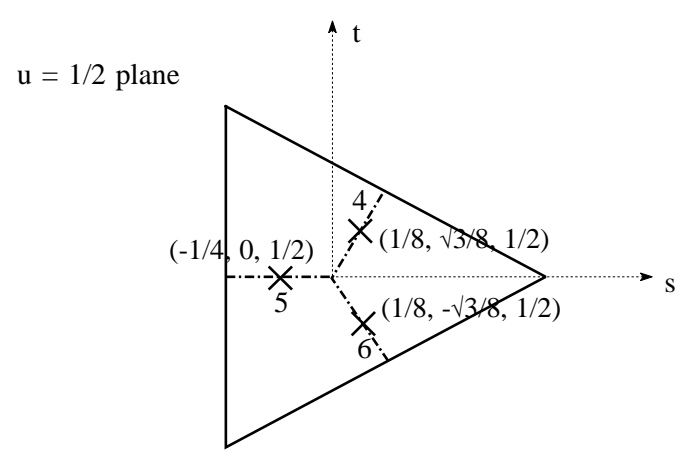

(b)

$$
\times \text { Integration points }
$$

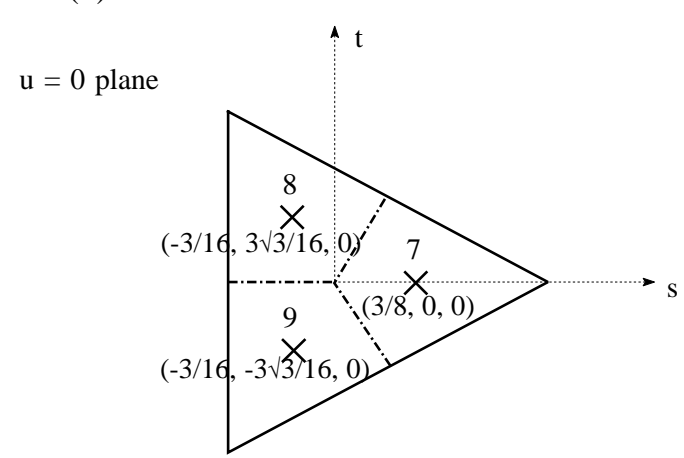

(c)

$$
\times \text { Integration points }
$$

Figure 9. BLP FV integration points in local coordinates. (a) $u=-\frac{1}{2}$, (b) $u=\frac{1}{2}$ and (c) $u=0$. 


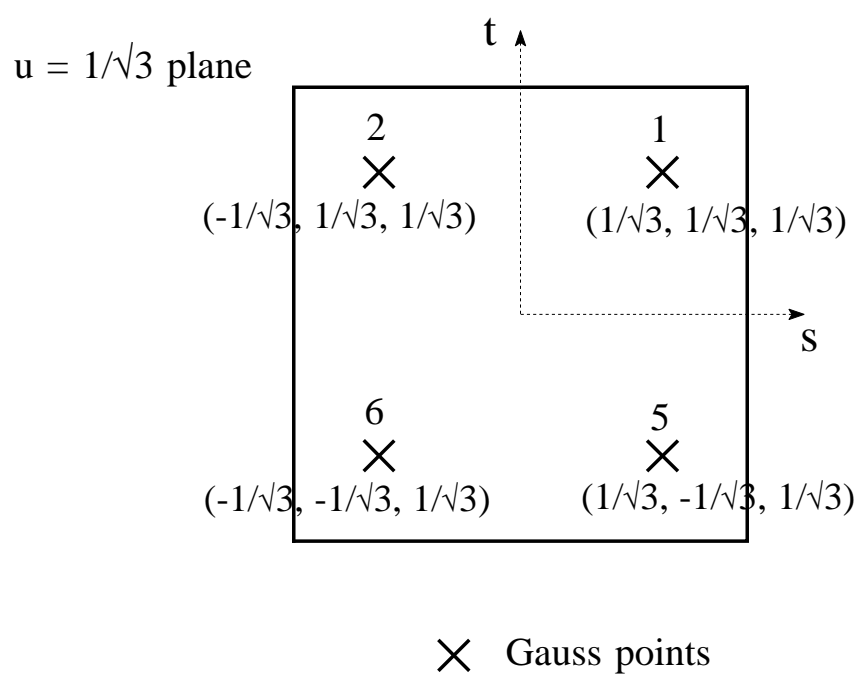

(a)

$$
\begin{aligned}
& \mathrm{u}=-1 / \sqrt{ } 3 \text { plane } \quad \mathrm{t}
\end{aligned}
$$

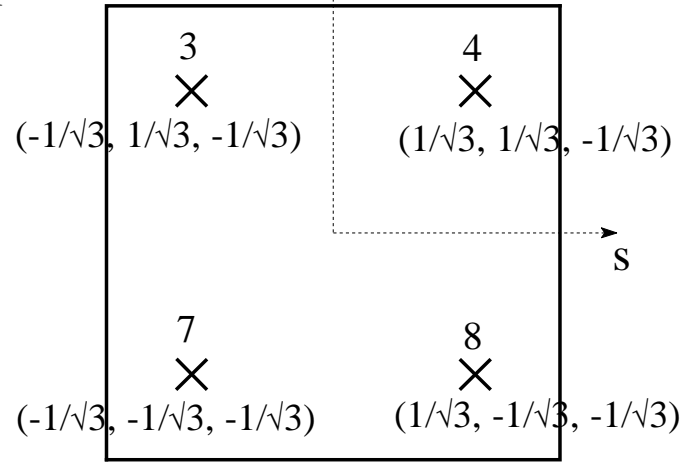

$$
\begin{aligned}
& \times \text { Gauss points }
\end{aligned}
$$

(b)

Figure 10. TLH Gauss points in local coordinates. (a) $u=1 / \sqrt{ } 3$ and (b) $u=-1 / \sqrt{ } 3$. 


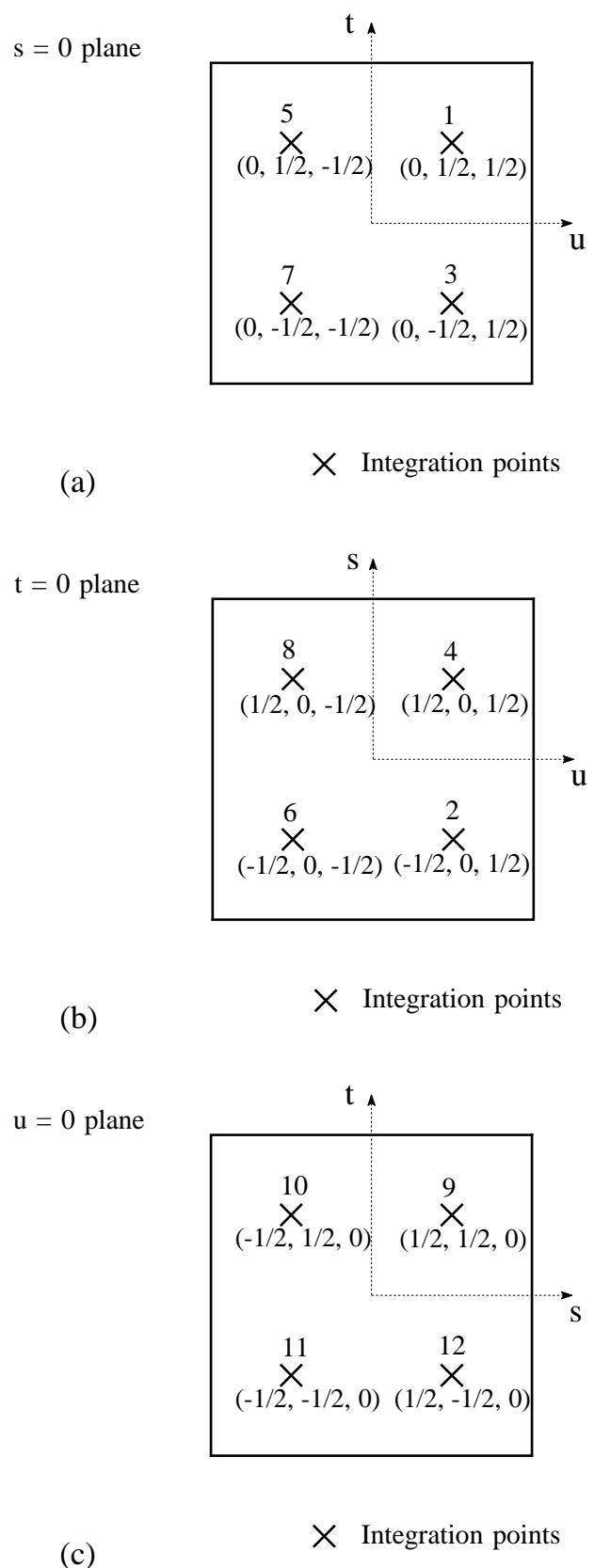

Figure 11. TLH FV integration points in local coordinates. (a) $u$, (b) $s$ and (c) $t$ planes. 


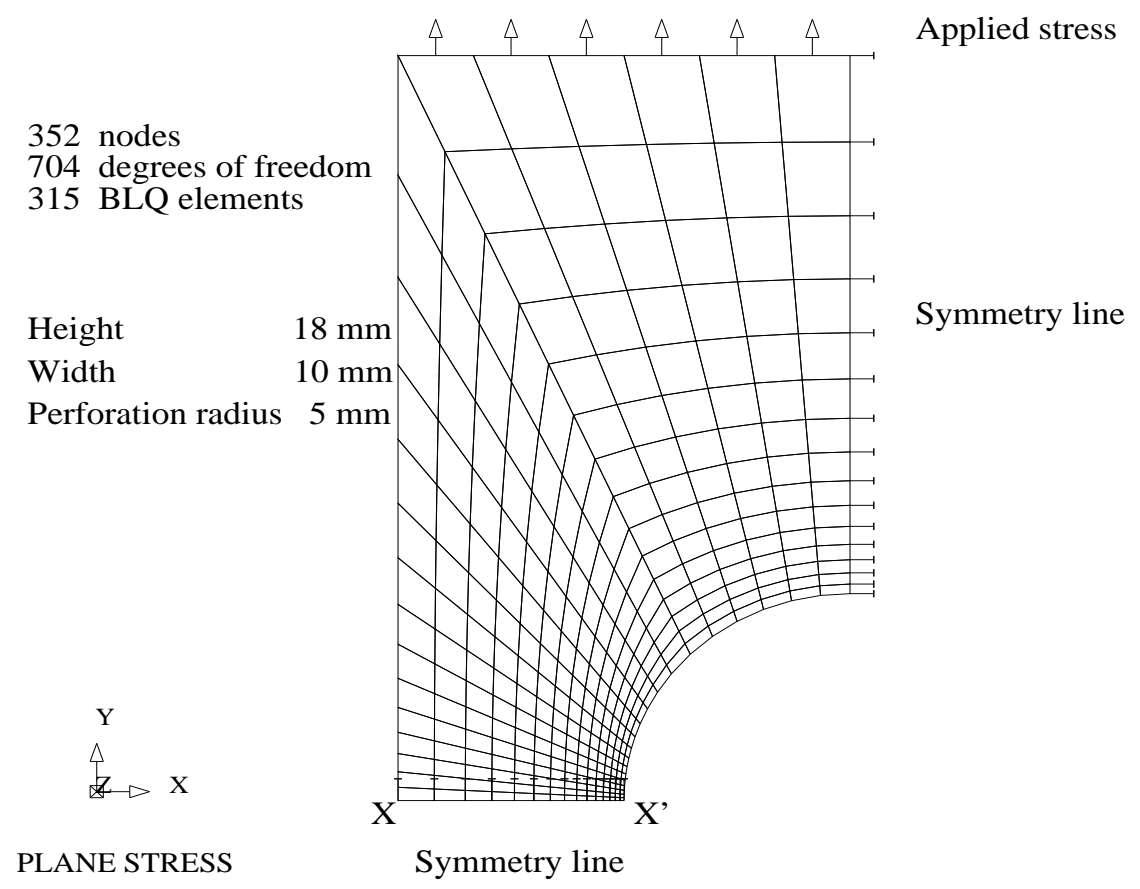

Figure 12. Schematic of a perforated tensile strip. 

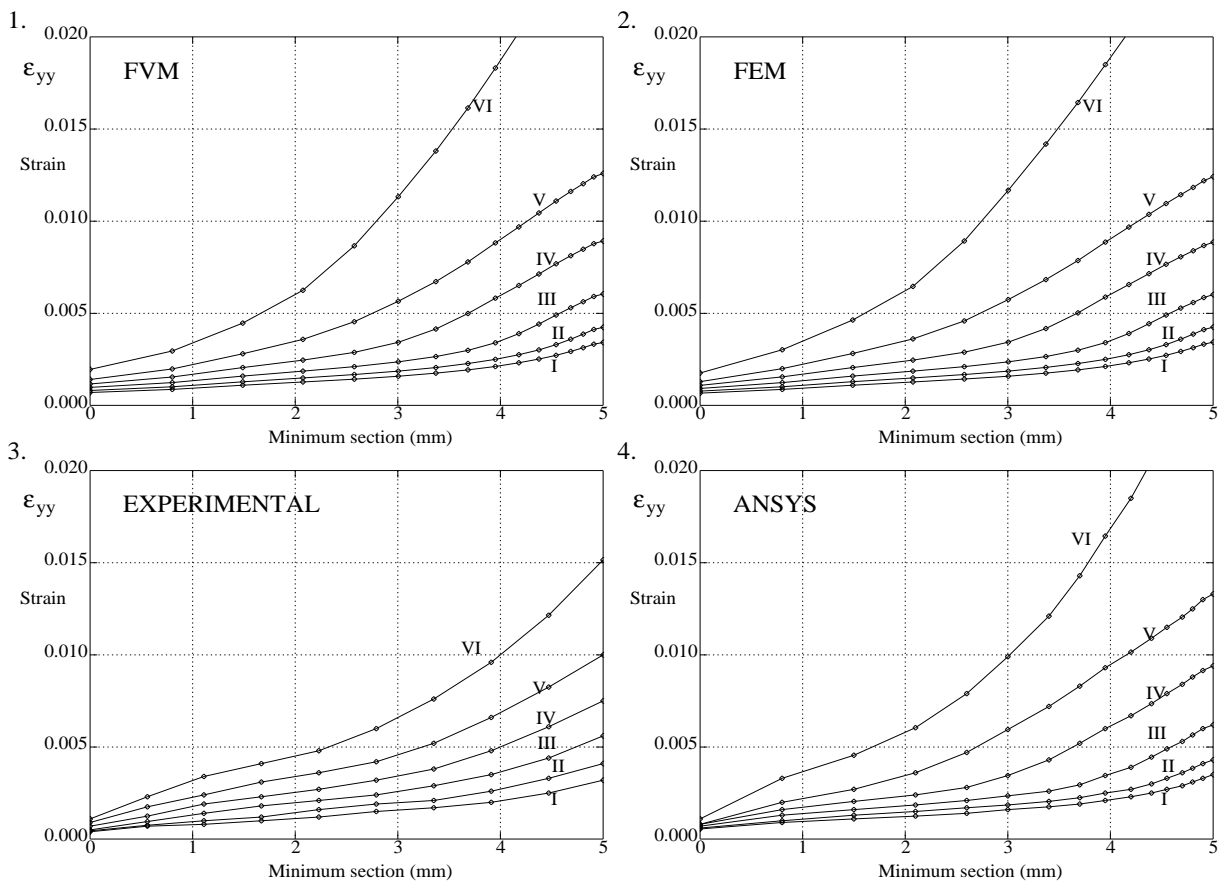

Figure 13. Predicted strain profiles for a perforated tensile strip. 

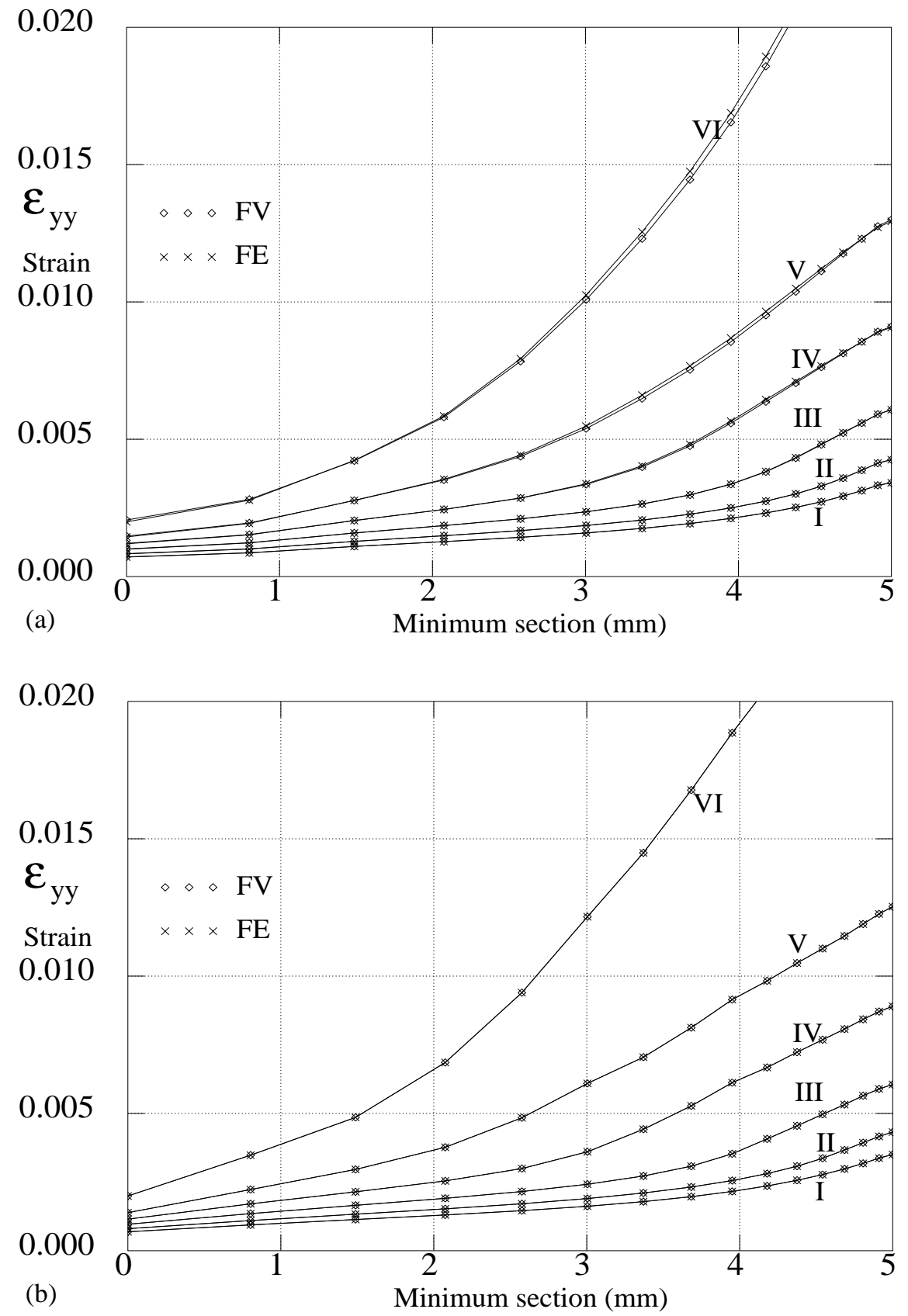

Figure 14. Comparison of the total strain for (a) BLQ and (b) CST elements. 


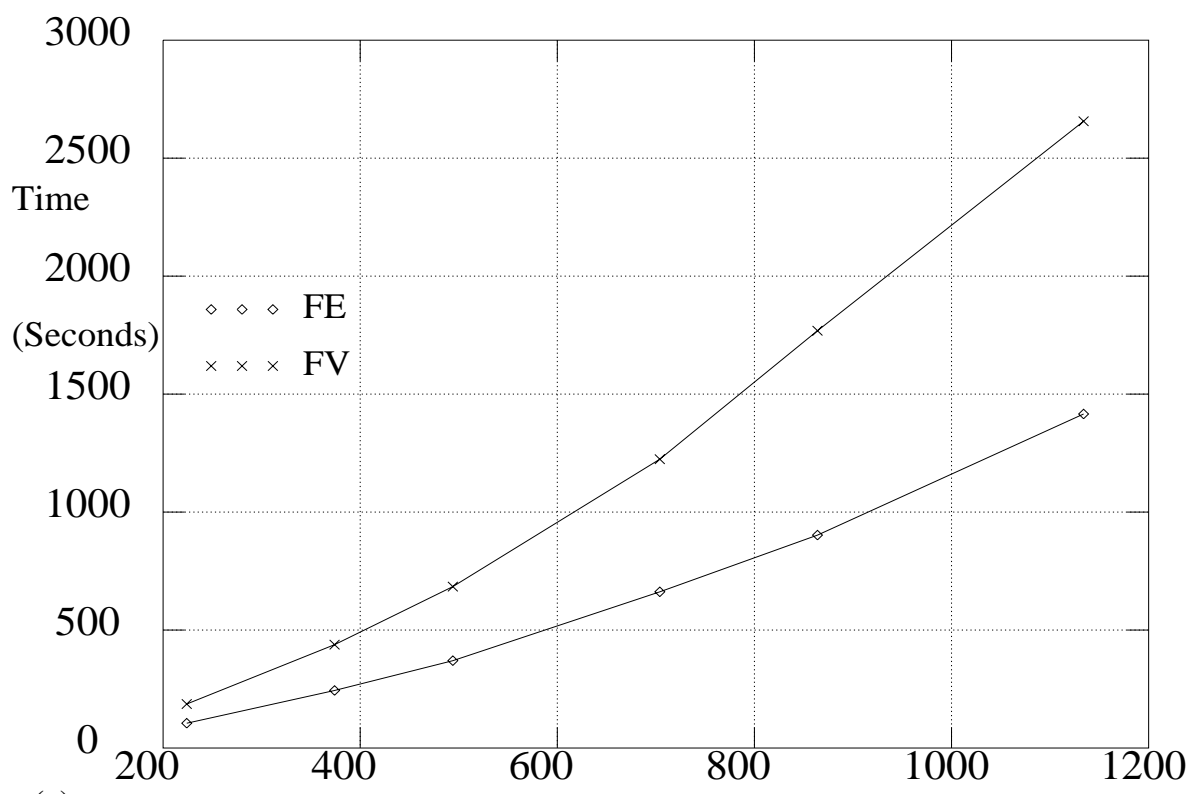

(a)

Degrees of freedom

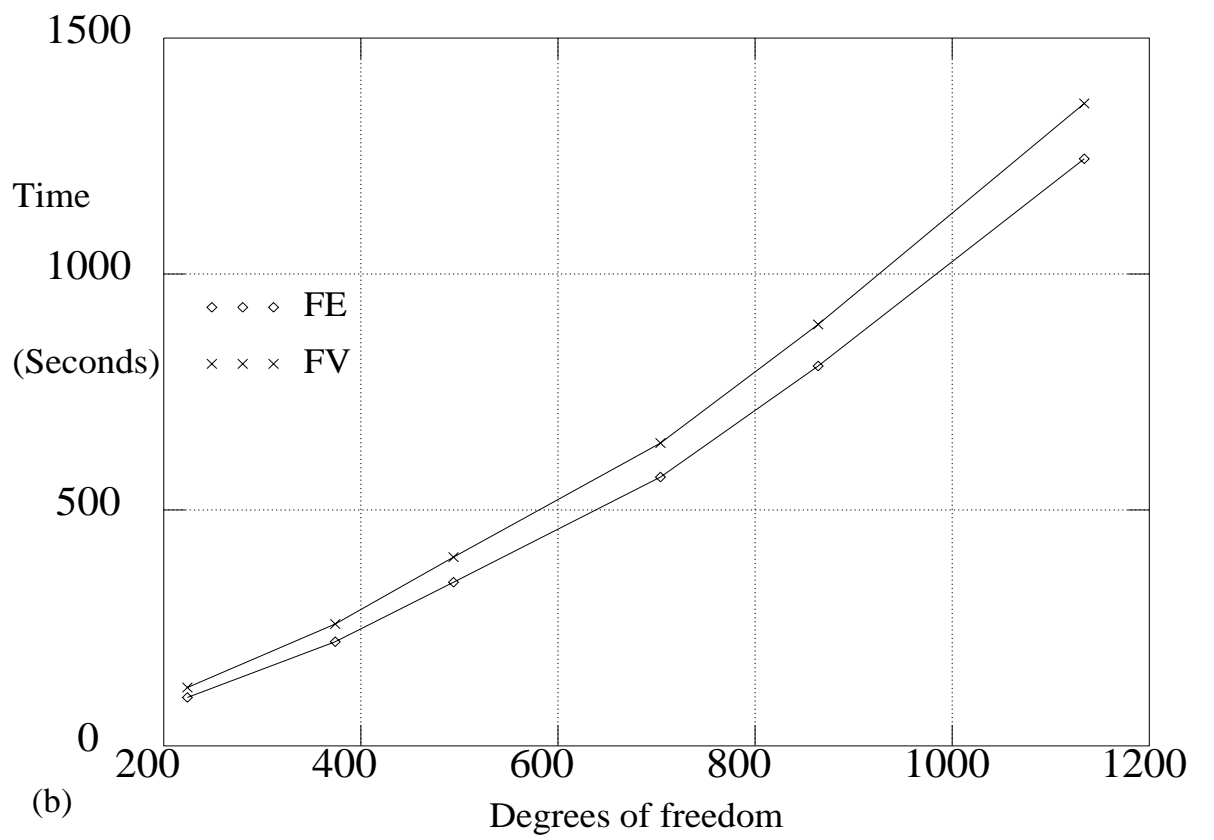

Figure 15. CPU times for (a) BLQ and (b) CST elements on a SPARC 4, 110MHz work station. 

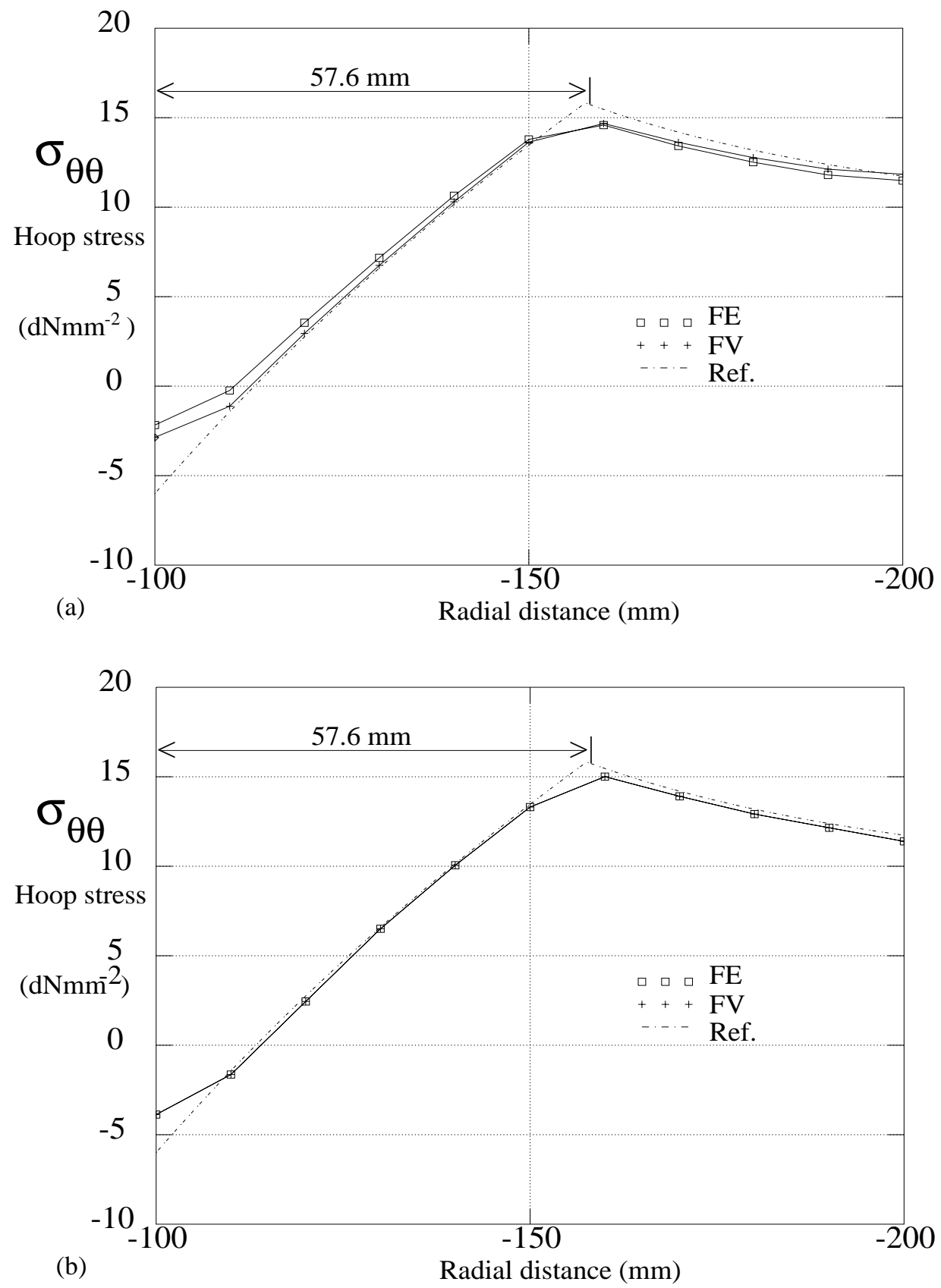

Figure 16. Stress profiles, (a) 950 TLH and (b) 4,800 LT elements. 

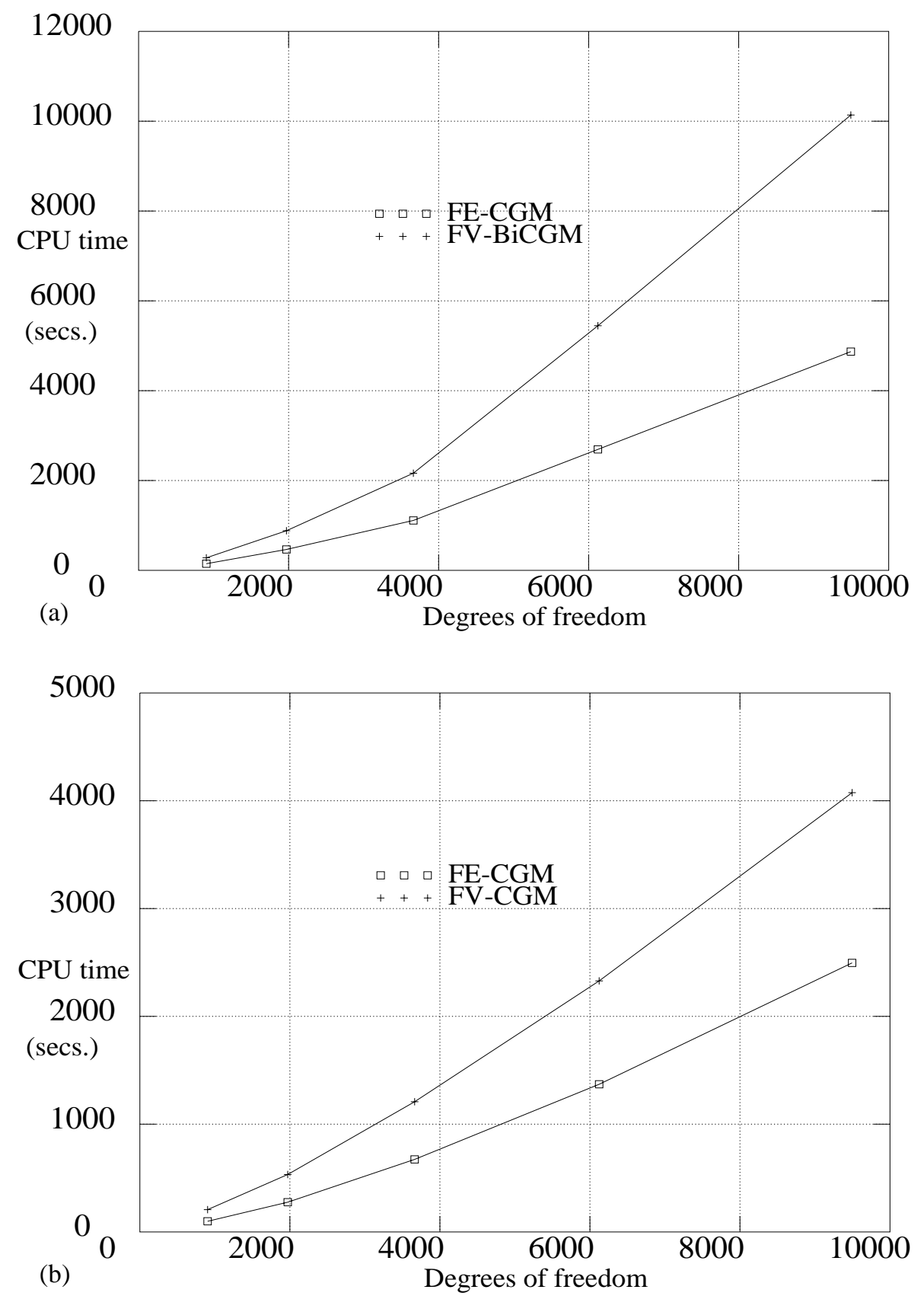

Figure 17. (a) TLH and (b) LT CPU times on a SPARC 4, 110MHz. 